University of Louisville

ThinkIR: The University of Louisville's Institutional Repository

Electronic Theses and Dissertations

$5-2021$

\title{
The role of Src in NSCLC cell and lung fibroblast migration on 3D cell-derived matrix.
}

Austin M Krueger

University of Louisville

Follow this and additional works at: https://ir.library.louisville.edu/etd

Part of the Pharmaceutics and Drug Design Commons

\section{Recommended Citation}

Krueger, Austin M, "The role of Src in NSCLC cell and lung fibroblast migration on 3D cell-derived matrix." (2021). Electronic Theses and Dissertations. Paper 3608.

https://doi.org/10.18297/etd/3608

This Master's Thesis is brought to you for free and open access by ThinkIR: The University of Louisville's Institutional Repository. It has been accepted for inclusion in Electronic Theses and Dissertations by an authorized administrator of ThinkIR: The University of Louisville's Institutional Repository. This title appears here courtesy of the author, who has retained all other copyrights. For more information, please contact thinkir@louisville.edu. 


\title{
CHARACTERIZING THE ROLE OF SRC IN NSCLC CELL AND LUNG FIBROBLAST MIGRATION ON 3D CELL-DERIVED MATRIX
}

\author{
By \\ Austin M. Krueger \\ B.S. Biology (Cellular and Molecular Physiology), University of Louisville, 2018
}

A Thesis submitted to the faculty of the School of Medicine of the University of Louisville in partial fulfillment of the requirements for the degree of

Master of Science in Pharmacology and Toxicology

\author{
Department of Pharmacology and Toxicology \\ University of Louisville \\ Louisville, Kentucky
}

May 2021 



\title{
CHARACTERIZING THE ROLE OF SRC IN NSCLC CELL AND LUNG FIBROBLAST MIGRATION ON 3D CELL-DERIVED MATRIX \\ By
}

\begin{abstract}
Austin M. Krueger
B.S. Biology (Cellular and Molecular Physiology), University of Louisville, 2018

A Thesis approved on

April 13, 2021
\end{abstract}

By the following Thesis committee:

Leah Siskind, Ph.D.

Levi Beverly, Ph.D.

Geoff Clark, Ph.D.

Howard Donninger, Ph.D.

Paula Bates, Ph.D.

Robert Mitchell, Ph.D. 


\section{DEDICATION}

I dedicate this dissertation to my parents and grandparents. Without their undying support and leadership, I would not have had the perseverance or heart to complete this. 


\section{ACKNOWLEDGEMENTS}

I would like to thank the past and present members of the Siskind and Beverly

labs. Without them, none of this work would have been possible. I would also like to thank my mentors, Leah Siskind and Levi Beverley, for supporting me throughout this project and pushing me to be the best scientist I could be. 


\section{ABSTRACT \\ THE ROLE OF SRC IN NSCLC CELL AND LUNG FIBROBLAST MIGRATION ON 3D CELL-DERIVED MATRIX}

Austin M. Krueger

April 13, 2021

Lung cancer is responsible for the most cancer deaths between males and females, with a $6 \%$ five-year survival rate. Cancer metastasis is one of the leading causes of lung cancer lethality. Our lab has developed a method to culture cells on cell-derived matrix (CDM) and study cell-ECM interactions. This study utilized multiple cell migration methods to investigate the migration of NSCLC cells and IMR90 lung fibroblasts, both together and independently, on 2D plastic and 3D CDM. Our results showed that inhibiting Src, a significant influencer of cell migration and member of integrin activation, blocked cancer cell migration on 2D and 3D CDM, regardless of interactions with fibroblasts or not. However, when IMR90 lung fibroblasts were treated with a Src inhibitor, migration decreased on 2D but not on 3D. These results suggest that cancer cells migrate in a Src-dependent manner on 3D CDM, but IMR90 lung fibroblasts do not. 
TABLE OF CONTENTS

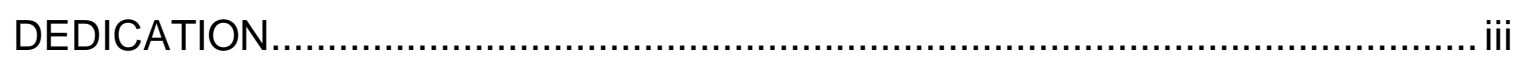

ACKNOWLEDGEMENTS …....................................................................... iv

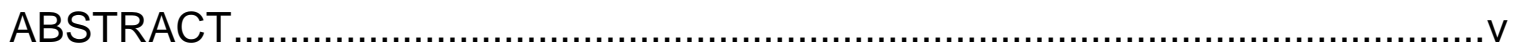

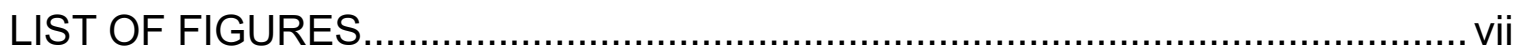

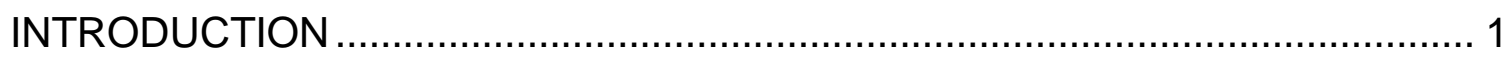

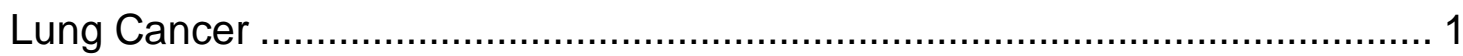

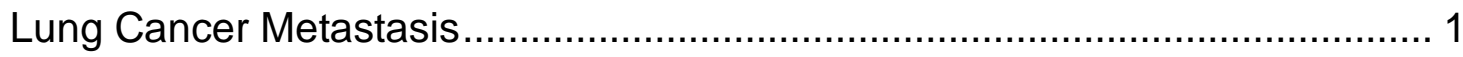

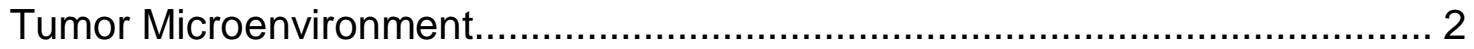

The Extracellular Matrix Environment ........................................................... 3

Epithelial to Mesenchymal Transition ………….......................................... 5

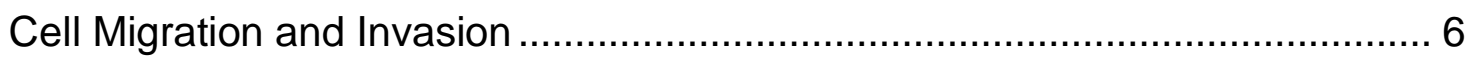

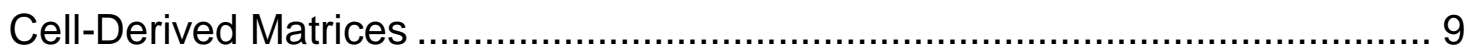

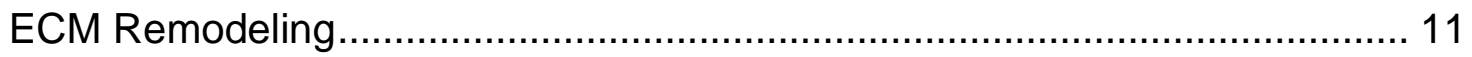

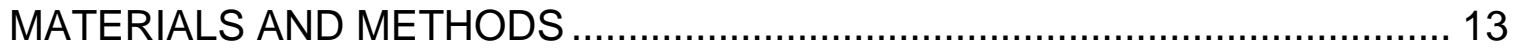

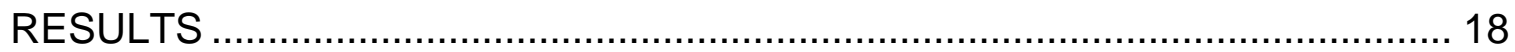

Non-small cell lung cancer cell migration increases on 3D cell-derived matrix.

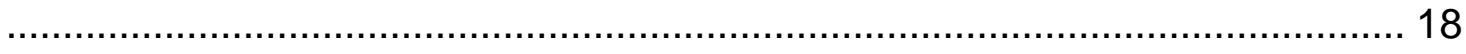

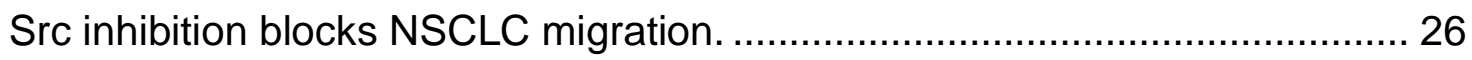

FAK inhibition alters NSCLC cell migration on 2D and 3D........................... 36

Co-cultured fibroblast migration is Src-independent on 3D............................ 46

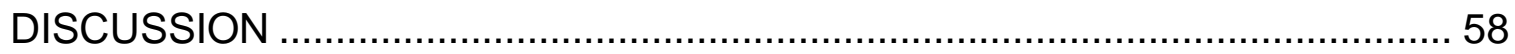

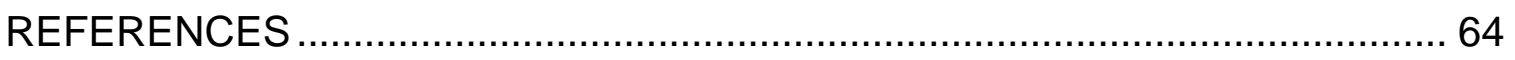

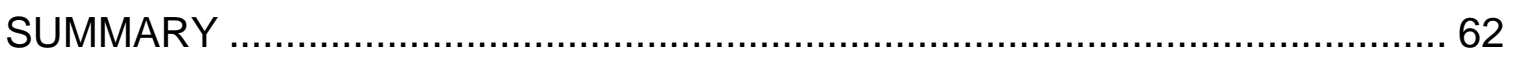

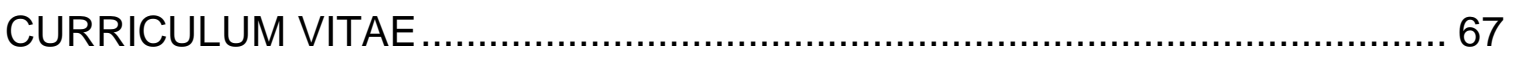




\section{LIST OF FIGURES}

Figure 1. Collective cell migration increases in Hop62 lung cancer cells on 3D. 19

Figure 2. Collective cell migration increases in H2030 lung cancer cells on 3D. 21

Figure 3. Collective cell migration of Hop62s increase on 3D regardless of the

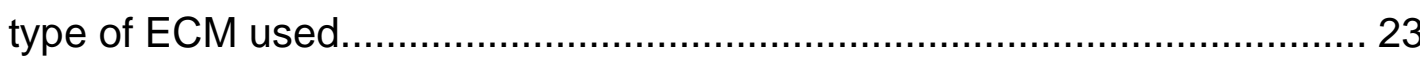

Figure 4. Relative wound closure is increased in A549 lung cancer cells on 3D. 25

Figure 5. Saracatinib inhibits Src phosphorylation in Hop62 lung cancer cells... 27

Figure 6. Saracatinib inhibits Src phosphorylation in A549 lung cancer cells..... 29

Figure 7. Saracatinib inhibits Src phosphorylation in H2030 lung cancer cells... 31

Figure 8. Saracatinib blocks the collective migration of Hop62 lung cancer cells.

Figure 9. Saracatinib blocks the collective migration of A549 lung cancer cells. 35 Figure 10. Saracatinib blocks the collective migration of H2030 lung cancer cells.

Figure 11. PF-573228 inhibits the autophosphorylation of FAK in Hop62 lung cancer cells. 
Figure 12. PF-573228 inhibits the autophosphorylation of FAK in A549 lung cancer cells.

Figure 13. PF-573228 decreases the collective cell migration of Hop62 lung cancer cells.

Figure 14. PF-573228 does not impact the collective cell migration of A549 lung cancer cells. 45

Figure 15. IMR90 lung fibroblasts co-cultured with A549 lung cancer cells migrate more on 3D CDM. 47

Figure 16. Saracatinib inhibits Src phosphorylation in IMR90 lung fibroblasts... 49 Figure 17. Saracatinib blocks the migration of IMR90 lung fibroblasts co-cultured with A549 lung cancer cells on 2D but not 3D. 51

Figure 18. Saracatinib blocks the migration of IMR90 lung fibroblasts co-cultured with Hop62 lung cancer cells on 2D but not 3D. 53

Figure 19. Saracatinib impacts the single cell migration of IMR90 lung fibroblasts on 2D. 55

Figure 20. Saracatinib does not impact the single cell migration of IMR90 lung fibroblasts on 3D. 57 


\section{INTRODUCTION}

\section{Lung Cancer}

Lung cancer is the leading cause of cancer deaths among males and females, accounting for the second most common type in both sexes in the United States [1]. Not only does lung cancer account for the most deaths, but it is also more fatal than colon, breast, and prostate combined [1]. There are two classifications of lung cancer: small cell and non-small cell [1, 2]. Small cell lung cancer accounts for an estimated $15 \%$ of lung cancer cases [2]. However, small cell lung cancer is more deadly than non-small cell lung cancer, with a 6\% 5-year survival rate [2]. Non-small cell lung cancer (NSCLC) is further classified into three groups: adenocarcinoma, squamous cell carcinoma, and large cell carcinoma. Adenocarcinoma is not only the most common cancer type worldwide but also the most common cancer type of NSCLC, accounting for more than $40 \%$ of incidences. It is evident that lung cancer poses a significant hurdle in cancer research today, but what is causing it?

\section{Lung Cancer Metastasis}

The National Cancer Institute defines metastasis as "the spread of cancer cells from the place where they first formed to another part of the body." The metastatic spread of cancer accounts for approximately $90 \%$ of cancer deaths [3]. 
Traditionally, a lung cancer patient may not observe significant symptoms until the disease has reached a later stage involving metastasis. If a patient is first diagnosed with lung cancer that metastasized to distant regions (regions other than the lung and tissues surrounding the lung), their chance to survive more than 5 years is $6 \%$ [1]. It is evident that most lung cancer deaths are due to metastasis and detecting the disease too late. Although several lung cancer prevention methods (screening, healthy diet/lifestyle, and avoiding smoking), there is still a considerable need to treat those with metastatic lung cancer.

Cancer metastasis can be divided into four separate stages: invasion and intravasation, circulation, extravasation, and metastatic colonization [3]. The first step in cancer metastasis is the cancer cells gaining enough mutations and tumor microenvironment (TME) cues to invade through the tissue's basement membrane [3]. Once they have invaded through the basement membrane, cancer cells can enter the patient's blood circulation. In animal models, approximately $0.01 \%$ of cancer cells survive in the host's blood circulation [4]. Because cancer cells are genetically unstable, the small percentage of cancer cells that remain in the host's circulation blood could extravasate. Following extravasation, the cancer cell may form a metastatic colony in another part of the body. So, how does one target cancer cell metastasis?

\section{Tumor Microenvironment}

The TME is an emerging topic in metastatic cancer research. Composed of numerous cell types, the TME plays a vital role in cancer cell survival, proliferation, and metastasis [5]. The primary cell types of the TME are cancer cells, epithelial 
cells, endothelial cells, fibroblasts, cancer-associated fibroblasts, and a plethora of immune cells [6]. Some non-cell components are the extracellular matrix (ECM), cytokines (growth factors, chemokines, etc.), and secreted proteins [6]. Targeting cancer metastasis has proven to be difficult. One possible reason is that many in vitro studies do not take the ECM into account. To investigate the interaction of NSCLC cells and the ECM, the entire TME must be considered.

Of the cellular components of the TME, fibroblasts perform critical functions in the interaction between cancer cells and the ECM. The major ECM secreting cell type in the body is fibroblasts [6]. Additionally, fibroblasts are responsible for secreting growth factors and other proteins involved in ECM remodeling [6]. An exciting topic in cancer research is investigating cancer-associated fibroblasts (CAFs). CAFs are commonly derived from tissue-resident fibroblasts and induced by components of the TME. Numerous studies show the different effects CAFs have on the TME as opposed to non-CAFs [7]. Among those differences are growth factor and cytokine secretions, as well as ECM remodeling [8]. Together, the impact of fibroblasts on the TME is vital in the ECM-cancer cell interplay.

\section{The Extracellular Matrix Environment}

The ECM contains many different compartments connecting the cell with the surrounding environment and impacting the cell on many different levels. The major structural components of the ECM are collagen, proteoglycans, laminin, and fibronectin [9]. Collagens are the most abundant proteins in the human body, comprising nearly $30 \%$ of tissue mass, including 28 different subtypes [10]. Collagens lay the foundation for the architecture of the ECM. Proteoglycans are 
composed of several subtypes. Perlecan, hyalecan, and small leucine-rich proteoglycan (SLRP) are the main proteoglycans that make up the ECM [9]. They play roles in collagen-fibronectin and collagen-collagen connections acting as the "glue" to hold the ECM together [11]. Laminins are made up of alpha, gamma, and delta chains comprising 16 different combinations. They are responsible for connecting the cell to the ECM [9]. Lastly, fibronectin is a multi-domain protein that interacts with the previous structural components discussed. Through these interactions, fibronectin provides connecting points for the cell and ECM. Each structural element of the ECM plays a unique role in the cancer cell-ECM interaction.

Embedded in the ECM are multiple types of ligands, including growth factors, chemokines, and other cytokines proteins, as well as proteases responsible for degrading the ECM [12]. Essential growth factors are tumor growth factor- $\beta$, interleukins, insulin-like growth factors, and epithelial growth factor [13]. Matrix metalloproteases play a critical role in ECM degradation, providing space for the cell to move through [14]. Each part of the ECM environment is involved in the ECM-cell interaction.

Aside from the external compartments, there is a copious number of components integrated into the cell membrane. Of these, integrins are one of the most critical transmembrane proteins involved in ECM-cell interactions. They contain an alpha and beta subunit, composing 24 known heterodimers with individual functions [15]. Binding to the ECM through collagen, laminin, and 
fibronectin, integrins provide a crucial cell connection to the ECM [16]. Their functions in cell invasion, adhesion, and migration are well documented.

AlphaV-Beta3 and Alpha5-Beta1 integrins, specifically, lead to focal adhesion kinase (FAK), Src family kinase (SFK) signaling activation, and eventual ERK, JUN, and Rac signaling activation [17]. When the integrins interact with the structural components and ligands of the ECM, the intracellular beta domain is activated by MRL proteins (MIG-10, RIAM, and Lamellipodin) [18] and recruits talin to promote actin polymerization and integrin clustering [16]. Integrin clustering ensures FAK and SFK activation. FAK/SFK and their downstream activation play one of the most critical roles in cell adhesion and movement.

\section{Epithelial to Mesenchymal Transition}

The Epithelial to Mesenchymal Transition (EMT) describes the transformation of epithelial-like cells to mesenchymal state [19]. An "epithelial-like" cell's rectangular shape will transition into a spindle-like, mesenchymal shape [20]. The transformation induces the cell to behave more stem-like, increasing its invasive and migratory phenotypes [19]. EMT contains a highly complex network of signaling to induce the mesenchymal states. The primary EMT inducers are TGF-beta, Wnt, Notch, hypoxia, PIK3, JAK/STAT, and integrin activation [21]. These pathways lead to the activation of critical EMT transcription factors: Snail 1, Slug, ZEB1/2, and Twist [21]. The transcription factors suppress the expression of crucial epithelial transition signalings, such as E-cadherin, occludins, claudins, and cytokeratins. N-cadherin, Vimentin, fibronectin, and MMPs are upregulated by the EMT transcription factors [19]. Not only do integrin-mediated FAK/SFK signaling 
and MMP activity play a critical role in cell-ECM interaction and remodeling, but they also are integral members of EMT.

The interaction between the cell and the extracellular matrix plays a vital role in cancer cell metastasis through external cues, such as ligand binding and integrin activation. Ligands embedded in the ECM and mutations acquired by cancer cells induce EMT through receptor-mediated activation. For example, TGFbeta, which is secreted by multiple cell types, induces EMT through the activation of SMAD2/3 and RAS/MAPK/ERK induction of EMT transcription factors [19]. Additionally, integrin activation induces FAK/SFK signaling to activate RAS, PI3K, STAT signaling, and EMT transcription factors [19]. Lastly, SNAIL1 and ZEB2 moderated the activation of MMPs responsible for ECM degradation [19]. How can one phenotypically show that alterations of these interactions influence cancer cell phenotype?

\section{Cell Migration and Invasion}

The ECM plays a crucial role in cell migration and invasion through alterations in signaling cascades related to EMT. Considering cancer metastasis steps, studying the invasion and migration of cancer cells in vitro can help researchers target cancer cell migration mechanisms from the primary tumor site. Four in vitro approaches to studying cancer cell migration are spheroid, scratch would, individual cell tracking, and trans-well assays:

1. A spheroid assay can be performed by culturing cancer cells in a nonadherent, round bottom 96-well plate long enough for the cells to form a spherical structure, the "spheroid" [22]. The spheroid is transferred to 
another plate, and the migration of cells from the spheroid can be monitored over time. This assay is a measure of collective cell migration. Using ImageJ, a software provided by the $\mathrm{NIH}$, the migration of cells can be quantified by defining the area of which the cells have migrated away from the core of the spheroid.

2. A scratch wound assay can be performed by creating a wound in a confluent cancer cell monolayer [23]. Once the wound is made, the collective migration of cells to close the wound can be monitored over time. Using ImageJ, a software provided by the $\mathrm{NIH}$, the migration of cells can be quantified by defining the area of which the cells have migrated into the wound. This may be referred to as the wound closure.

3. One may also monitor individual cell migration by individually tracking cancer cells' velocity and directionality over time [24]. This may be done by adding a low concentration, single-cell suspension, to a cell culture plate. The cells are monitored over time using live-cell imaging and utilizing available cell tracking softwares.

4. A trans-well assay is performed by culturing cancer cells in nutrient-free media on top of a Boyden chamber. The Boyden chamber contains small pores wide enough for single cells to fit through [25]. On the other side of the pore is media containing nutrients and other factors to attract the cancer cells [25]. One can then quantify the number of cells that migrated through the pores over time by quantifying the number of cells attached to the bottom of the cell culture plate. 
Together, these four assays allow researchers to study cancer cell migration from many different angles.

A critical step in cancer metastasis is cancer cells breaking through the basement membrane and out of the primary tumor site to other sites in the body. Invasion assays are very similar to migration assays except for one added component: Matrigel. Matrigel is a gel-like substance at room temperature that can act as a basement membrane and is comparable to embryonic basement membranes [26]. In-depth proteomic analysis shows the key components of Matrigel are laminin-1, collagen IV, entactin, and heparan sulfate proteoglycan, which provide both structural support and comparable signal transduction to the embryonic basement membrane/ECM [27]. With Matrigel, researchers can modify migration assays into invasion assays:

1. Adding Matrigel to the non-adherent well containing the spheroid encapsulates the spheroid with the gel-like substance [22]. Cells then invade into the gel surrounding the spheroid as well as away from the spheroid itself. Using Image J, a software provided by the $\mathrm{NIH}$, the migration of cells can be quantified by defining the area of which the cells have migrated away from the core of the spheroid.

2. In a scratch wound assay, the addition of a thin monolayer of Matrigel to the top of the cells overlays the wound made. This allows the cells to invade into the wound [28]. Using ImageJ, a software provided by the $\mathrm{NIH}$, the migration of cells can be quantified by defining the area of which the cells have migrated into the wound. 
3. By creating a 1:1 ratio of low concentrated, single-cell suspension and Matrigel, one can add the mixture to a cell culture plate. Because the cells are encapsulated in Matrigel, cells are forced to invade through the gel-like substance [24]. The cells are monitored over time using live-cell imaging and utilizing available cell tracking softwares.

4. Adding a layer of Matrigel to the Boyden chamber pores would force the cells to invade through the Matrigel to reach the nutrient-filled media below [29]. One can then quantify the number of cells that migrated through the pores over time by quantifying the number of cells attached to the bottom of the cell culture plate.

Using these techniques, researchers can study cancer cell invasion in vitro.

The World Health Organization reported approximately 10 million people die from cancer each year. As one of the leading causes of death worldwide, cancer research is crucial in finding better treatments for cancer patients. Because cancer metastasis is responsible for a worse 5-year survival rate, investigating cancer cell migration and invasion may provide helpful information to design drugs to target cancer metastasis. There are multiple assays to study cancer cell migration and invasion, each with its own positive and negative characteristics. Choosing which assay to use will depend on a researcher's available technology and time.

\section{Cell-Derived Matrices}

Knowing that the ECM plays a pivotal role in cancer cell migration and invasion, how do researchers apply this concept to the previously discussed 
migration and invasion assays? Utilizing fibroblasts' ability to produce a surplus of extracellular matrix, one can culture fibroblasts long enough to deposit a layer of ECM on a cell culture plate. Once the fibroblasts decellularized from the plate, a layer of ECM is left behind. Researchers can then seed cells on top of the ECM layer and study the cell's interaction with the ECM [30].

The most apparent difference between cells cultured on 3D CDM and 2D plastic is the cell phenotype. To perform various migration and invasions assays using 3D CDM, one would perform the assay the same way except that the cells are cultured on 3D CDM. Cells naturally shaped like a cube in standard 2D culture methods appear more elongated and spindle-like in a 3D CDM environment [28]. With this phenotype, researchers studied the signaling differences in cells cultured in a 3D CDM system. Remarkably, the differences were incredibly obtuse. EMT markers were increased in cells cultured on 3D CDM, and the proliferation, migration, and invasion of cancer cells were significantly different [30]. Specifically, epithelial markers were decreased, and mesenchymal markers were increased in cells cultured on 3D CDM [30]. FAK/SFK signaling is also affected. Phenotypically, proliferation is slightly decreased while migration and invasion are increased [30]. Culturing cells on 2D plastic as opposed to 3D CDM yields different phenotypes and cancer cell signaling.

Cell-derived matrices (CDMs) impact cells in many ways, and the technique is well documented and highly reproducible [28] [30] [31]. It is postulated that culturing cells on ECM is a more physiologically relevant model 
for cancer research in vitro. Using 3D CDM culture techniques may yield more consistent in vitro results.

\section{ECM Remodeling}

Velocity and displacement increased significantly in NSCLC cells cultured on 3D CDM [30]. An important distinction between 3D CDM and 2D plastic is the physical appearance. Expectedly, 2D plastic looks flat and appears smooth. 3D CDM seems more rigid and gelatinous. Using light microscopy, one can see the collagen fibers of the ECM are aligned in a web-like network. As a whole, the CDM may seem unorganized; however, the CDM's rigidity is what provides directionality to the cells. Cell-derived ECM provides a road network for cells cultured on CDM. Cancer cells react to the framework the CDM provides.

Fibroblasts are the central regulators of the tumor ECM environment [32]. They are responsible for secreting soluble actors, ECM remodeling, metabolic effects, and immune crosstalk and secrete many different soluble secretory factors, such as VEG-F and TGF-Beta [32]. They remodel the ECM through matrix crosslinking, proteolysis, and matrix production [32]. Metabolic effects include lactate shuffling (providing lactate to cancer cells dependent on such for energy) and amino acid shuffling [32]. Lastly, fibroblasts interact with surrounding immune cells through TGF-Beta and IL-6 activation and CCL2 production. The interaction between cancer cells and fibroblasts is synergistic, meaning fibroblasts can prime cancer cells for invasion/migration, and cancer cells can induce an activated phenotype of fibroblasts. 
It is thought that there are three resting states of fibroblasts: resting, normal-activated, and fibrosis/cancer-associated [33]. Fibroblasts are typically found in a resting form where surrounding stimuli can activate them to normalactivated fibroblasts. In their resting state, fibroblasts are spindle-shaped, do not migrate, produce ECM, or secrete soluble factors [33]. Normal-activated fibroblasts are identified primarily through alpha-SMA and vimentin expression [33]. This transition is reversible through genetic reprogramming and induction of apoptosis. Normal-activated fibroblasts play a considerable role in wound healing and fibrosis. From the normal activated state, fibroblasts can transition into fibrosis- or cancer-associated fibroblasts through epigenetic regulation. One key identifiable marker is fibroblast activation protein expression. The critical difference between the two states is that cancer-associated fibroblasts proliferate more, secrete more growth factors, and do not produce as much ECM [33]. Once fibroblasts are in this state, they cannot revert to normal-activated fibroblasts.

It is apparent that taking into account multiple members of the TME is increasingly vital in studying cancer cell migration and invasion. Our study aimed to investigate the interaction between cancer cells, fibroblasts, and the ECM independently and co-dependently. Our results suggest that NSCLC cells and fibroblasts migrate through different migration mechanisms on 3D cell-derived matrix, where they migrate through similar mechanisms on 2D. This information is critical in elucidating the methods by which the TME impacts cancer cell migration. 


\section{MATERIALS AND METHODS}

\section{Cell culture}

Human normal fetal lung fibroblast cell lines WI38, IMR90, and CCL-201 were purchased from American Type Culture Collection (ATCC) and maintained in Minimum Essential Medium Alpha modification (MEM- $\alpha$ ) supplemented with LGln, ribo- and deoxyribonucleosides (Fisher),10\% fetal bovine serum (Invitrogen) and 1\% antibiotic/antimycotic (Sigma). Human mesenchymal cell lines (WI38 and IMR90) were performed with early passage cells $(p<8)$ in the same culturing medium, MEMa, described previously. A549, H2009, and H2030 human adenocarcinoma cells were purchased from ATCC and were cultured in RPMI medium supplemented with 10\% FBS and 1\% antibiotic. All cell lines were maintained at $37^{\circ} \mathrm{C}$ and $5 \% \mathrm{CO}^{2}$, unless otherwise stated.

\section{Extracellular Matrix Preparation}

Extracellular matrix preparation was performed as previously described [30]. Briefly, IMR90, WI38, or CCL-201 lung fibroblasts were plated at confluence in matrix 
growth medium (MEM-a, 10\% FBS, $1 \%$ antibiotic). On the second, fourth, and sixth days, medium was supplemented with $50 \mu \mathrm{g} / \mathrm{mL}$ L-Ascorbic acid (Sigma) to promote matrix deposition. On the eighth day, wells were washed with phosphate buffered saline (PBS), and cells were lysed with a detergent $(20 \mathrm{mM} \mathrm{NH} 4 \mathrm{OH}$, $0.05 \%$ Triton-X-100) for $1-3$ minute incubation at $37^{\circ} \mathrm{C}$ until complete cell lysis was observed. The resulting cell-derived matrices were gently washed three times with PBS and then incubated with Dnase1 $(10 \mathrm{U} / \mathrm{mL})$ for 30-60 minutes to remove residual DNA. The matrices were then washed twice in PBS and either used immediately or stored at $4^{\circ} \mathrm{C}$ in PBS supplemented with $1 \%$ antibiotic for up to 4 months.

\section{Microscopy}

Live-cell time-lapse phase and fluorescence microscopy were performed using a Keyence All-in-One Fluorescence Microscope (BZ-X series).

\section{Scratch Wound Migration Assay}

Lung cancer cells were plated at confluence and serum-starved overnight following attachment. A micropipette tip was used to create a scratch in the cell monolayer, and pictures were captured at the indicated time points. 3D invasion assays were plated using a WI38 derived matrix that had not been supplemented with ascorbic acid during the growth. Ascorbic acid supplementation or use of IMR90 fibroblast matrices results in a matrix that was too thick to scratch reliably. Images were captured at the indicated timepoints. Results were quantified using the area measurement of imageJ, comparing the wound closure at the time of 
the image to the initial wound. Averages of at least three images were captured for each time point, and at least two replicates were used for each experiment.

\section{Spheroid Preparation}

Lung cancer cells were culture under normal conditions on a Corning ultralow attachment 96 well plate (Sigma) at $1 \times 10^{4}$ cells per well for 4 days to allow small, rounded spheroids. Spheroids were observed under microscopy and transferred to matrix plates with a micropipette for further migration studies.

\section{Western Blot}

Cells and tissue samples were harvested in CHAPS lysis buffer (1\% CHAPS detergent, $150 \mathrm{mM} \mathrm{NaCl}, 50 \mathrm{mM}$ Tris $\mathrm{pH} 7,5 \mathrm{mM}$ EDTA) supplemented with complete protease inhibitor and PhosSTOP phosphatase inhibitor cocktail tablets (Sigma-Aldrich, St. Louis, MO). Total protein $(40 \mu \mathrm{g})$ was heated at $95^{\circ} \mathrm{C}$ for 5 minutes and separated by $4-12 \%$ SDS-polyacrylamide gel. Blots were transferred to PVDF membranes for 120 minutes at a constant voltage of $100 \mathrm{~V}$ in $4^{\circ} \mathrm{C}$. Membranes were blocked in 5\% milk (w/v) in Tris-buffered saline Tween-20 (TBS-T) at $24^{\circ} \mathrm{C}$ for 1 hour. The membranes were incubated with the primary antibody in $5 \%$ milk TBS-T overnight at $4^{\circ} \mathrm{C}$. A list of antibodies used for analysis is presented in the supplementary material. 


\begin{tabular}{|c|c|c|c|}
\hline Antibody & Host & Catalog & Distributor \\
\hline \hline Src & Rabbit & $\# 2108$ & Cell Signaling Technologies \\
P-Src Family (Tyr416) & Rabbit & $\# 2101$ & Cell Signaling Technologies \\
FAK (D2R2E) & Rabbit & $\# 13009$ & Cell Signaling Technologies \\
P-FAK (Tyr397) (D20B1) & Rabbit & $\# 8556$ & Cell Signaling Technologies \\
GAPDH (D16H11) & Rabbit & $\# 5174$ & Cell Signaling Technologies \\
Rabbit IgG HRP & Goat & $\# 7074$ & Cell Signaling Technologies \\
\hline
\end{tabular}

\section{Table 1. Antibodies.}




\section{Statistics}

Student's t-tests were used for comparisons between two groups. A one-way ANOVA with Tukey's test for multiple comparisons was used to determine statistical significance for multiple groups, unless otherwise stated. Statistical significance was considered for $\mathrm{P}$-value $<0.05$. 


\section{RESULTS}

\section{Non-small cell lung cancer cell migration increases on 3D cell-derived matrix.}

We have previously shown that cancer cell signaling and proliferation change on the 3D cell-derived matrix [30]. To investigate how culturing cells on 3D cellderived matrix influences cell migration, we examined spheroid spreading on $2 \mathrm{D}$ plastic and 3D IMR90-derived matrix. Hop62 non-small cell lung cancer (NSCLC) cell spheroids were cultured on 2D plastic and 3D IMR90-derived matrix for 48 hours (Figure 1a). The migrational area of the spheroids was measured and quantified via ImageJ. Hop62 spheroids cultured on 3D migrated significantly more than the spheroids cultured on 2D (Figure 1b). 


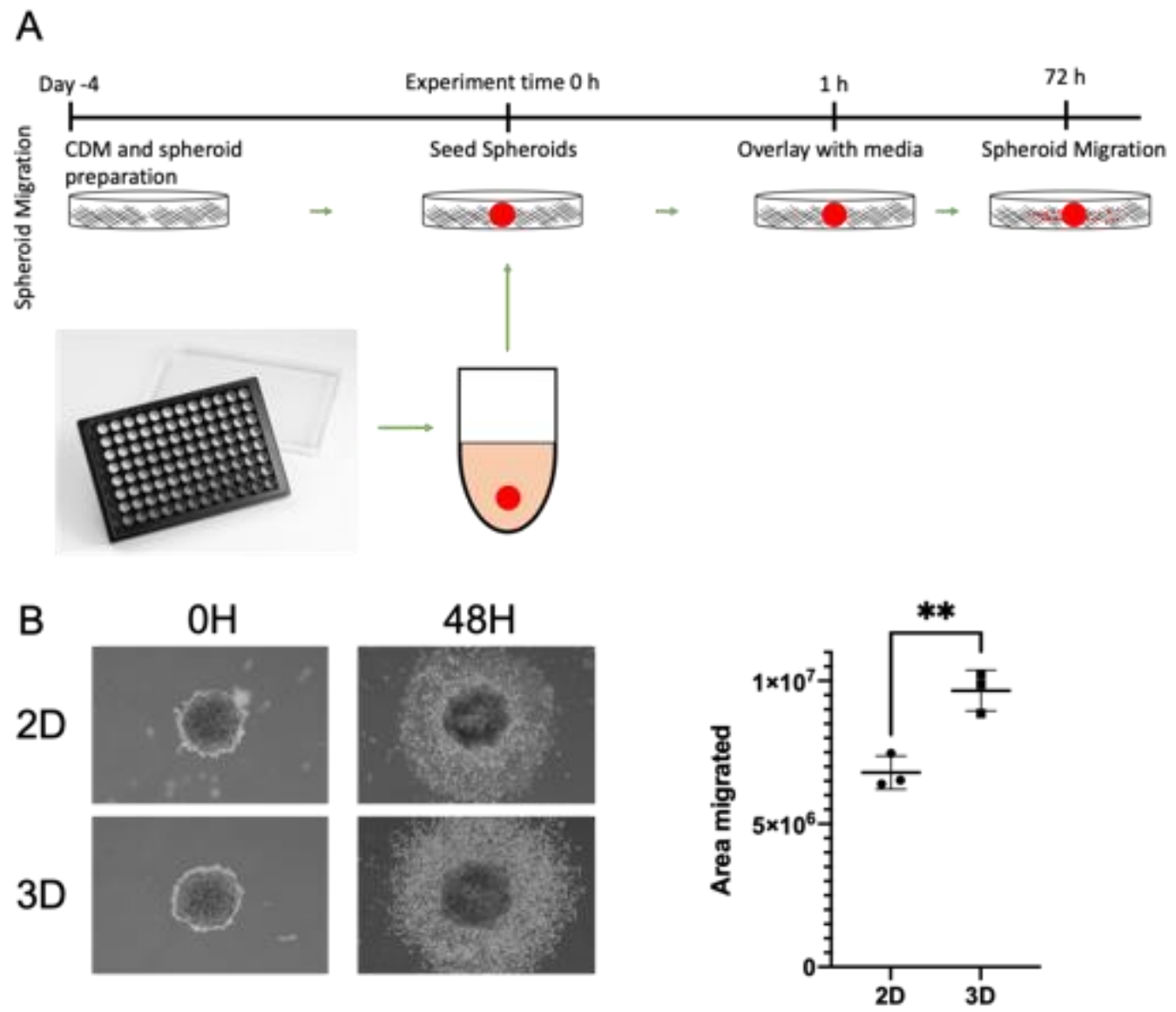

Figure 1. Collective cell migration increases in Hop62 lung cancer cells on 3D.

Hop62 spheroids were grown for four days and transferred to 2D plastic, and 3D IMR90 derived ECM. A) Schematic of Spheroid Migration Assay. B) Images were captured at 0 and 48 hours after initial plating. C) Quantification of total migrated area relative to the area of the spheroid. Statistical analysis performed by unpaired T-test was performed, ${ }^{* *} p<0.01$; Data presented as mean \pm S.E.M. 
H2030 NSCLC cell spheroids were cultured on 2D plastic and 3D IMR90-derived matrix for 48 hours (Figure 2a). The migrational area of the spheroids was measured and quantified via ImageJ. H2030 spheroids cultured on 3D migrated significantly more than the spheroids cultured on 2D (Figure 2b). 
A

$\mathrm{OH}$

$48 \mathrm{H}$

2D


3D
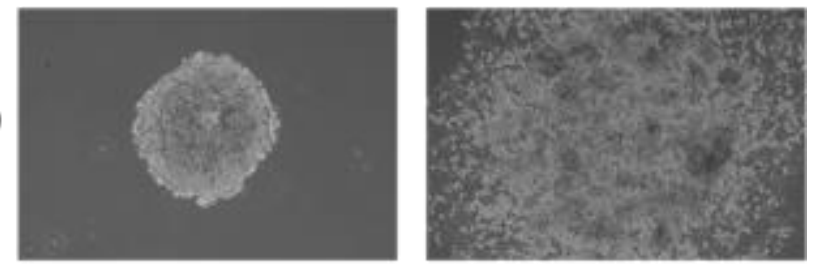

B

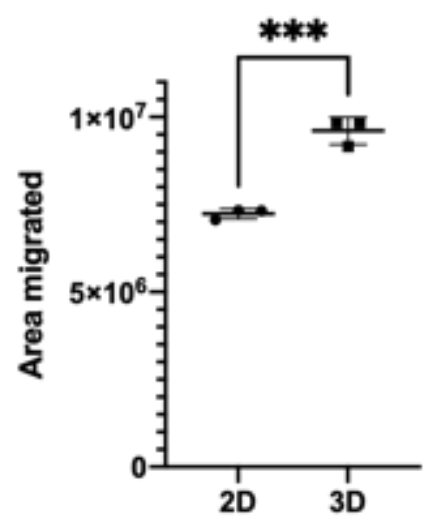

Figure 2. Collective cell migration increases in $\mathrm{H} 2030$ lung cancer cells on 3D.

H2030 spheroids were grown for four days and transferred to 2D plastic, and 3D IMR90 derived ECM. A) Images were captured at 0 and 48 hours after initial plating. B) Quantification of total migrated area relative to the area of the spheroid. Statistical analysis performed by unpaired T-test was performed, *** $\mathrm{p}<0.005$; Data presented as mean \pm S.E.M. 
Because migration was increased on 3D, we wanted to investigate whether the type of matrix mattered. Hop62 spheroids were cultured on 2D, 3D IMR90 derived matrix, and 3D CCL-201 (adult lung fibroblast) derived matrix for 48 hours (Figure 3a). The migrational area of the spheroids was measured and quantified via ImageJ. Hop62 spheroid migration increased on 3D compared to $2 \mathrm{D}$ regardless of the matrix type (Figure $3 b$ ). 
A
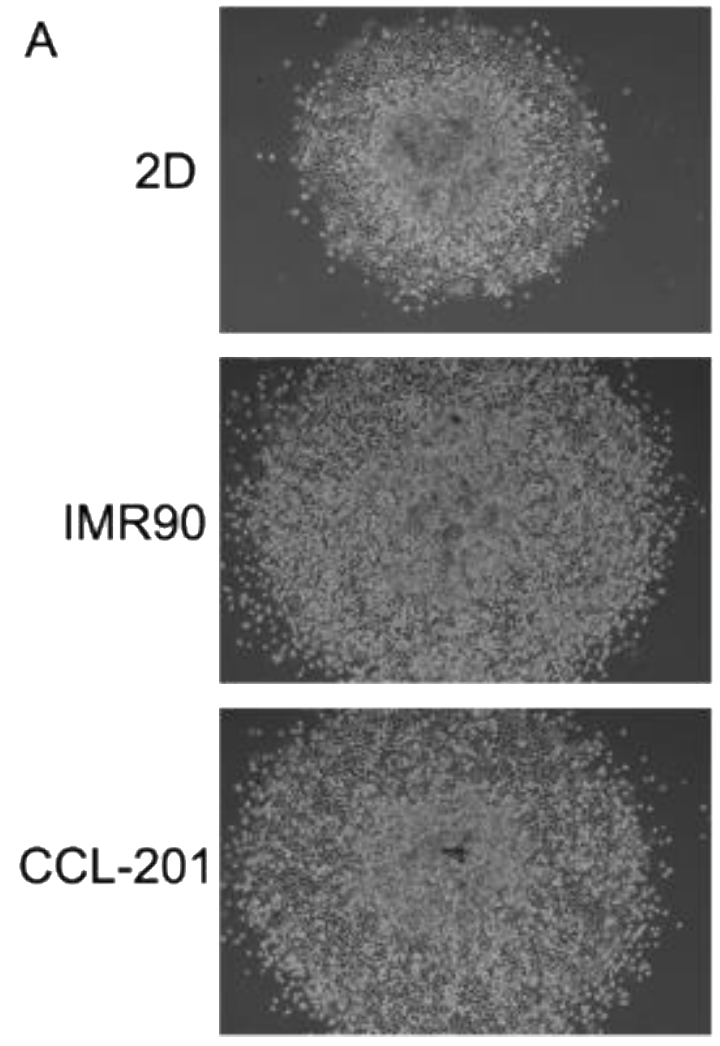

B Hop62 2DviMR90vCCL-201 Spheroid Assay

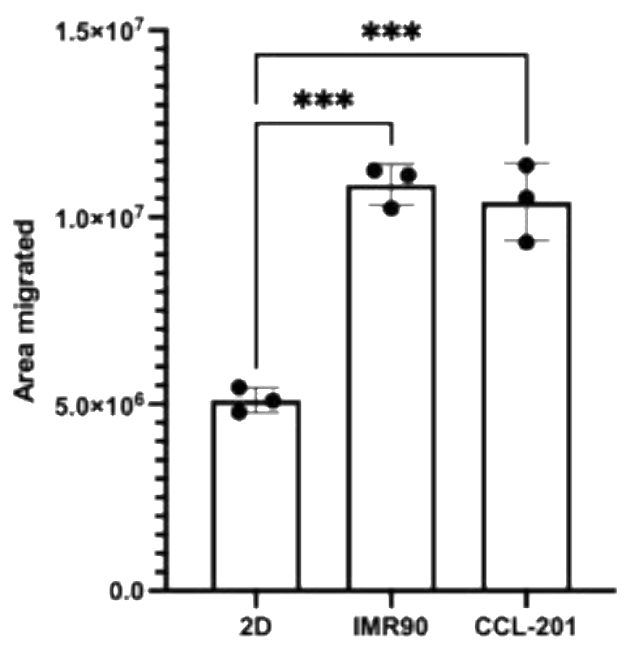

Figure 3. Collective cell migration of Hop62s increase on 3D regardless of the type of ECM used.

Hop62 spheroids were grown for four days and transferred to 2D plastic and multiple 3D cell-derived ECM. B) Images were captured 48 hours after initial plating. A) Quantification of total migrated area relative to the area of the spheroid. Statistical analysis performed by 1-way ANOVA was performed with multiple comparisons; ${ }^{* * *} p<0.005$; Data presented as mean \pm S.E.M. 
To investigate whether the phenotype was dependent on the migrational as performed, a scratch wound migration assay was performed with A549 NSCLC cells on 2D plastic and 3D IMR90 derived matrix (Figure 4a). The relative wound closures were recorded at 6, 24, and 48 hours and quantified via ImageJ. The wound closure was significantly increased on $3 \mathrm{D}$ compared to $2 \mathrm{D}$ at all time points (Figure 4b). These data suggest that NSCLC cells migrate more on 3D compared to 2D. 



Figure 4. Relative wound closure is increased in A549 lung cancer cells on 3D.

A549 cancer cells are grown on 2D or 3D CDM at confluence, and a scratch wound is created. The excellent area is then either overlaid with complete media.

A) Images were captured at 0, 6, 24, and 48 hours. B) Quantification of the relative wound area. Statistical analysis performed by unpaired t-test was performed, ${ }^{* *} p<0.01,{ }^{* *} p<0.005$; Data presented as mean $\pm S$.E.M. 


\section{Src inhibition blocks NSCLC migration.}

It had previously been shown that integrin signaling is altered in A549 cancer cells cultured on 2D compared to 3D cell-derived matrix [30]. It is well documented that Src plays a pivotal role in integrin signaling and cell migration $[17,34,35]$. To investigate the role of Src, an Src inhibitor (Saracatinib) was used to inhibit Src phosphorylation in Hop62, H2030, and A549 NSCLC cells. To determine the concertation of Saracatinib that was not lethal to Hop62 cells, an Alamar Blue cell viability assay with varying concentrations $(0-20 \mu \mathrm{M})$ of Saracatinib at 24, 48, and 72 hours was performed (Figure 5a). Next, Hop62 cells were cultured on 2D and 3D with varying concentrations of Saracatinib (0$10 \mu \mathrm{M})$ to determine which concertation inhibits Src phosphorylation (Figure 5b). In Hop62 cells, saracatinib significantly inhibited Src phosphorylation at the $10 \mu \mathrm{M}$ range on $2 \mathrm{D}$ and the $5-10 \mu \mathrm{M}$ range on $3 \mathrm{D}$ (Figure $5 \mathrm{~b}$ ). 


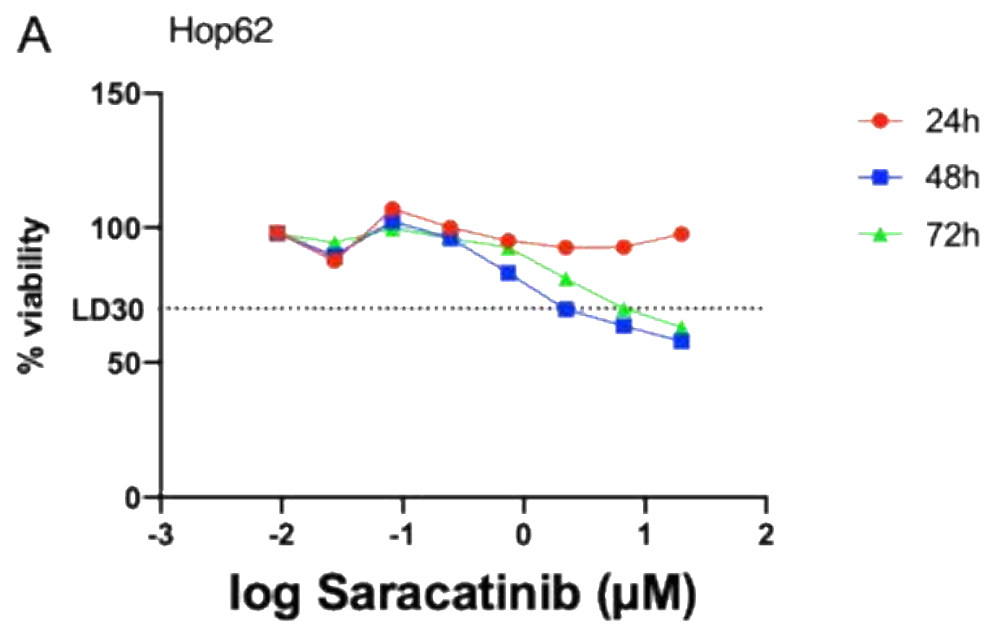

B

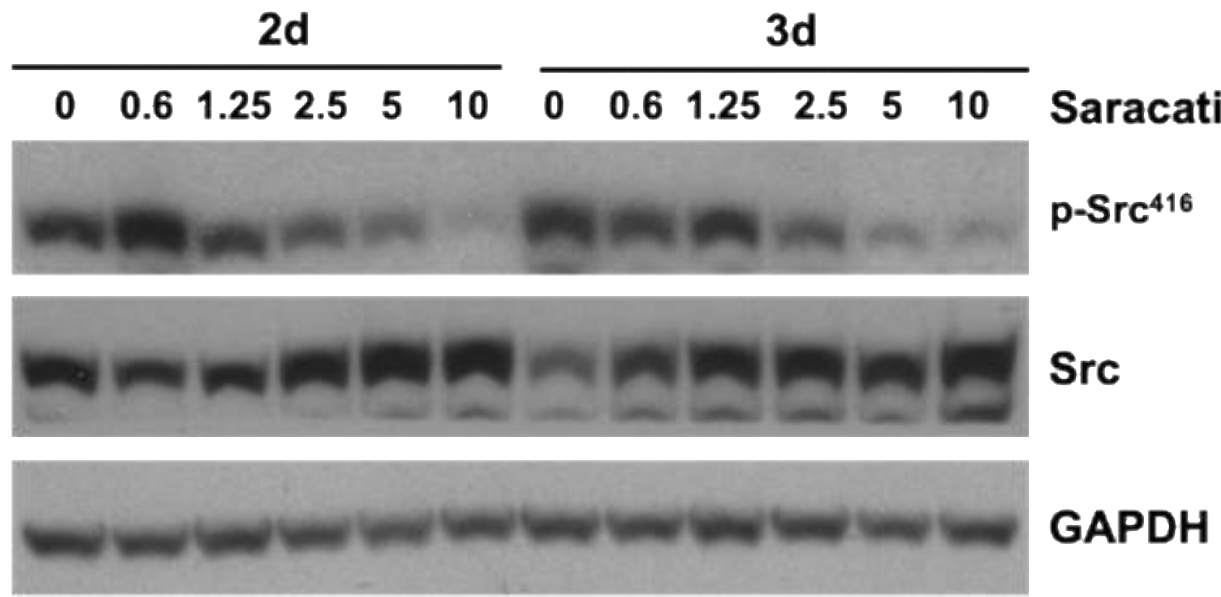

Figure 5. Saracatinib inhibits Src phosphorylation in Hop62 lung cancer cells.

Hop62 cells were treated with varying concentrations of Saracatinib. A) Alamar Blue readings were recorded 24, 48, and 72 hours following treatment. B) Hop62 cells were plated on 2D, and 3D IMR90 derived matrix. The cells were treated with varying concentrations of Saracatinib and collected 72 hours following treatment. Western blot analysis of the extracts was probed for p-src, src, and GAPDH. 
To determine the concertation of Saracatinib that was not lethal to A549 cells, an Alamar Blue cell viability assay with varying concentrations $(0-20 \mu \mathrm{M})$ of Saracatinib at 24, 48, and 72 hours was performed (Figure 6a). Next, A549 cells were cultured on 2D and 3D with varying concentrations of Saracatinib $(0-10 \mu \mathrm{M})$ to determine which concertation inhibits Src phosphorylation (Figure 6b).

Saracatinib significantly inhibited Src phosphorylation from the $0.6-10 \mu \mathrm{M}$ range in A549 cells on both 2D and 3D (Figure 6b). 


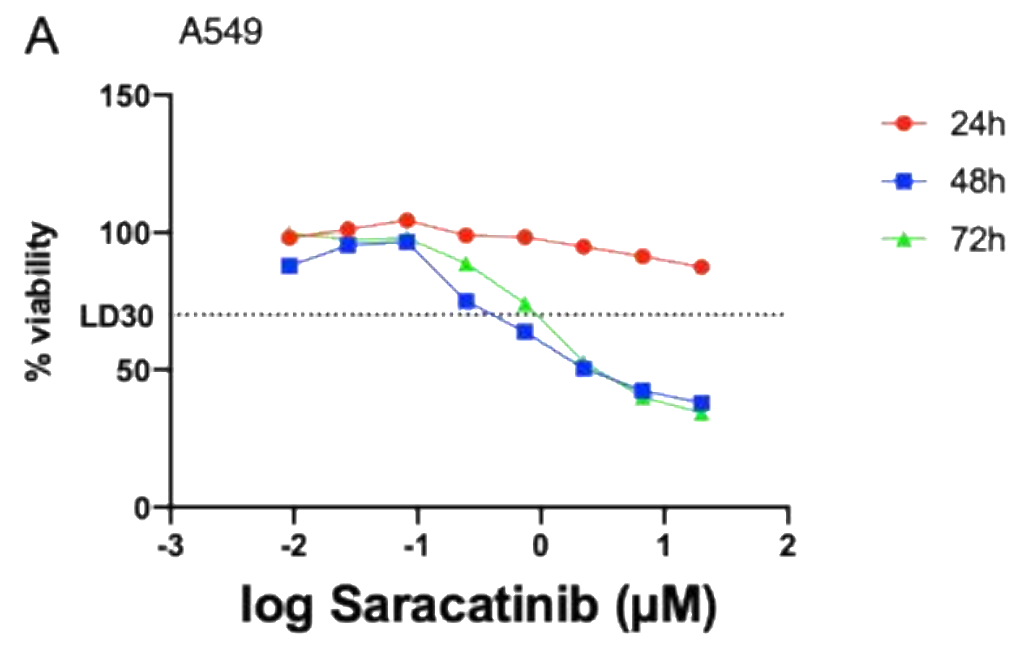

B

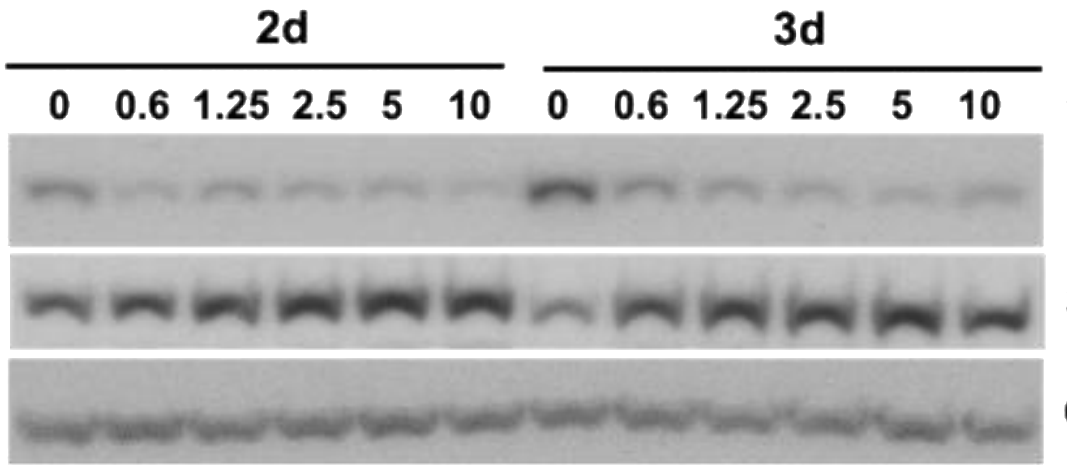

Saracatinib $(\mu \mathrm{M})$ p-Src ${ }^{416}$

Src

\section{GAPDH}

Figure 6. Saracatinib inhibits Src phosphorylation in A549 lung cancer cells.

A549 cells were treated with varying concentrations of Saracatinib. A) Alamar Blue readings were recorded 24,48 , and 72 hours following treatment. B) A549 cells were plated on 2D, and 3D IMR90 derived matrix. The cells were treated with varying concentrations of Saracatinib and collected 72 hours following treatment. Western blot analysis of the extracts was probed for p-src, src, and GAPDH. 
To determine the concertation of Saracatinib that was not lethal to $\mathrm{H} 2030$ cells, an Alamar Blue cell viability assay with varying concentrations $(0-20 \mu \mathrm{M})$ of Saracatinib at 24, 48, and 72 hours was performed (Figure 7a). Next, H2030 cells were cultured on 2D and 3D with varying concentrations of Saracatinib (0$10 \mu \mathrm{M})$ to determine which concertation inhibits Src phosphorylation (Figure $7 \mathrm{~b}$ ). In H2030 cells, saracatinib significantly inhibited Src phosphorylation at the 1.25$10 \mu \mathrm{M}$ range on $2 \mathrm{D}$ and the $2.5-10 \mu \mathrm{M}$ range on $3 \mathrm{D}$ (Figure $7 \mathrm{~b}$ ). 


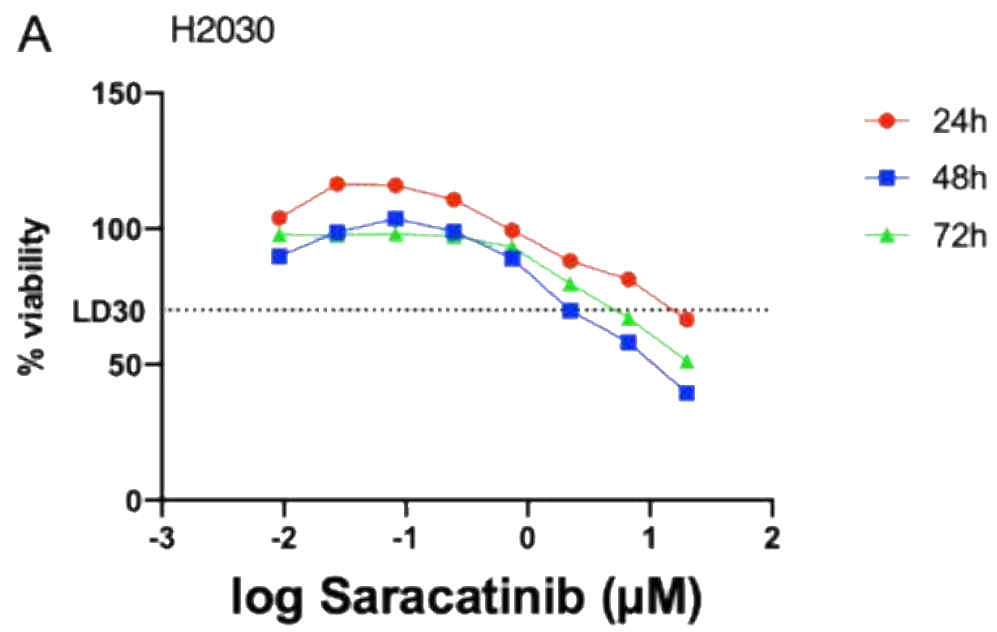

B 2d 3d

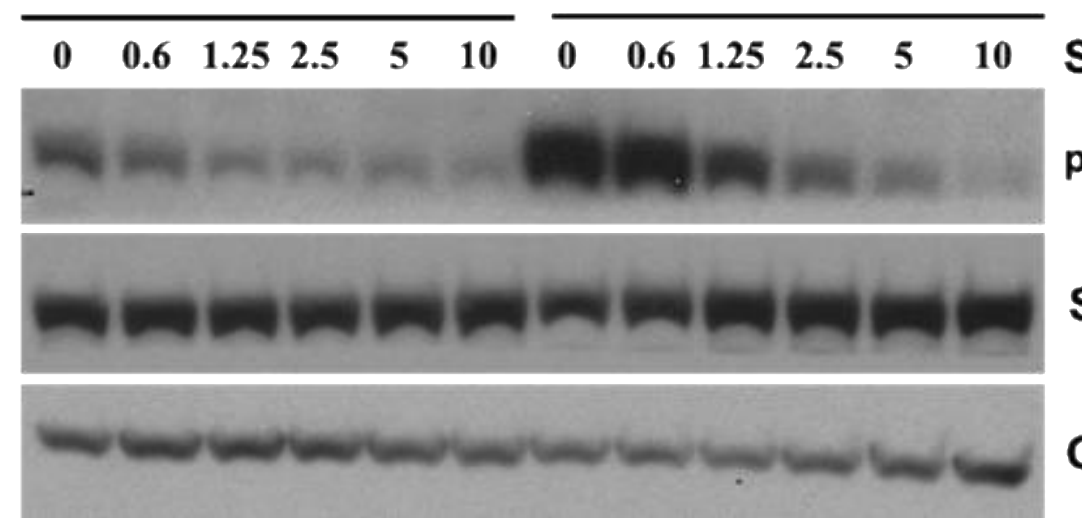

Saracatinib $(\mu \mathrm{M})$

p-Src ${ }^{416}$

Src

GAPDH

Figure 7. Saracatinib inhibits Src phosphorylation in H2030 lung cancer cells.

H2030 cells were treated with varying concentrations of Saracatinib. A) Alamar Blue readings were recorded 24, 48, and 72 hours following treatment. B) H2030 cells were plated on 2D, and 3D IMR90 derived matrix. The cells were treated with varying concentrations of Saracatinib and collected 72 hours following treatment. Western blot analysis of the extracts was probed for p-src, src, and GAPDH. 
To assess the inhibition of Src's effect on cancer cell migration, spheroid assays were performed on 2D and 3D with multiple lung cancer cell lines and treated with Saracatinib or not. Hop62 cell spheroids, treated with $5 \mu \mathrm{M}$ of Saracatinib or a DMSO vehicle control, were cultured on 2D plastic and 3D IMR90-derived matrix for 48 hours (Figure 8a). The migrational area of the spheroids was measured and quantified via ImageJ. Hop62 spheroids treated with Saracatinib migrated significantly less on 2D and 3D compared to the vehicle control (Figure 8b). 
A

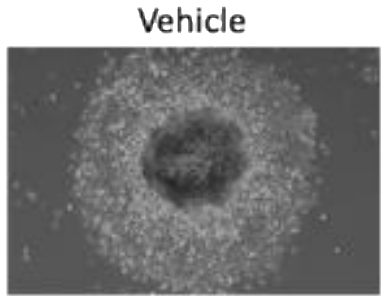

$5 \mu \mathrm{M}$ Saracatinib

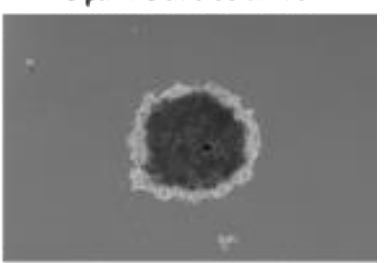

3D

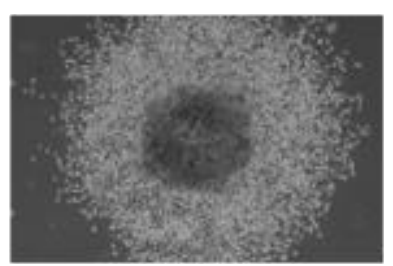

B Hop62 Saracatinib Spheroid Assay

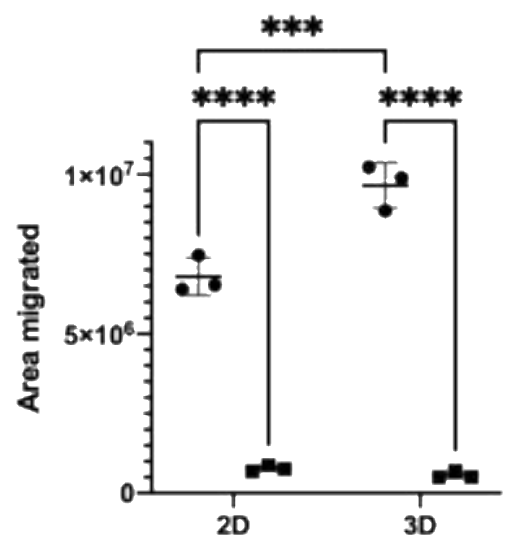

- Vehicle

- $5 \mu \mathrm{M}$ Saractinib

Figure 8. Saracatinib blocks the collective migration of Hop62 lung cancer cells.

Hop62 spheroids were grown for four days and transferred to 2D plastic, and 3D IMR90 derived ECM. The spheroids were treated with Saracatinib $(5 \mu \mathrm{M})$ or Vehicle (DMSO). A) Images were captured 48 hours after initial plating. B) Quantification of total migrated area relative to the area of the spheroid. Statistical analysis performed by 2-way ANOVA was performed with multiple comparisons, ${ }^{* * *} p<0.005,{ }^{* * * *} p<0.001$; Data presented as mean $\pm S . E . M$. 
A549 cell spheroids, treated with $5 \mu \mathrm{M}$ of Saracatinib or a DMSO vehicle control, were cultured on 2D plastic and 3D IMR90-derived matrix for 48 hours (Figure 9a). The migrational area of the spheroids was measured and quantified via ImageJ. A549 spheroids treated with Saracatinib migrated significantly less on 2D and 3D compared to the vehicle control (Figure 9b). 


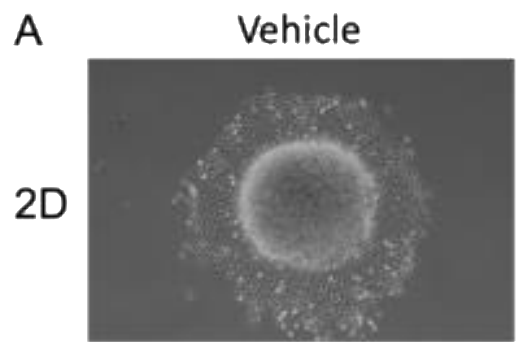

$5 \mu \mathrm{M}$ Saracatinib

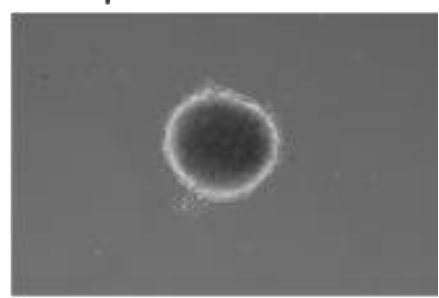

$3 \mathrm{D}$
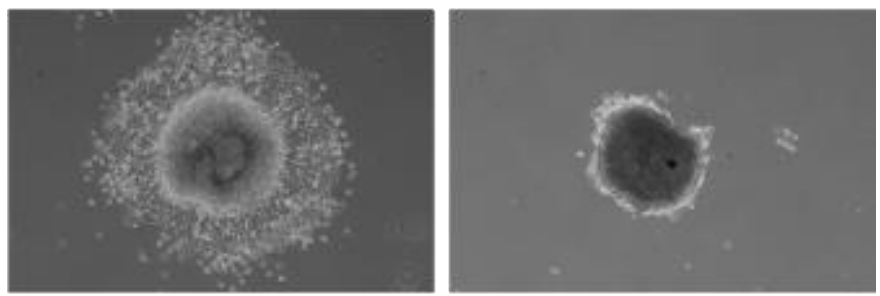

B A549 Saracatinib Spheroid Assay

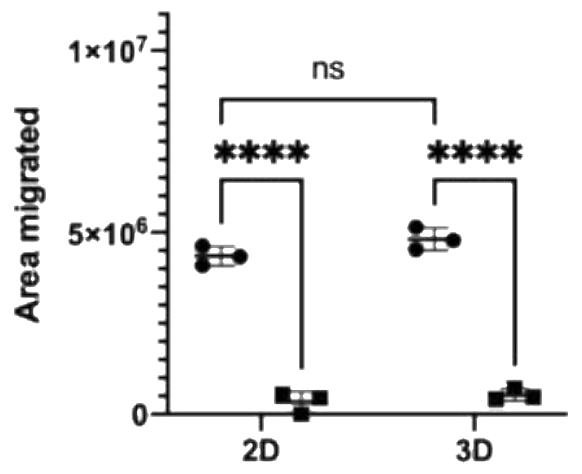

- Vehicle

- $5 \mu \mathrm{M}$ Saractinib

Figure 9. Saracatinib blocks the collective migration of A549 lung cancer cells.

A549 spheroids were grown for four days and transferred to 2D plastic, and 3D IMR90 derived ECM. The spheroids were treated with Saracatinib $(5 \mu \mathrm{M})$ or Vehicle (DMSO). A) Images were captured 48 hours after initial plating. B) Quantification of total migrated area relative to the area of the spheroid. Statistical analysis performed by 2-way ANOVA was performed with multiple comparisons, ${ }^{* * * *} p<0.001$; Data presented as mean \pm S.E.M. 
H2030 cell spheroids, treated with $5 \mu \mathrm{M}$ of Saracatinib or a DMSO vehicle control, were cultured on 2D plastic and 3D IMR90-derived matrix for 48 hours (Figure 8a). The migrational area of the spheroids was measured and quantified via ImageJ. H2030 spheroids treated with Saracatinib migrated significantly less on 2D and 3D compared to the vehicle control (Figure 10b).

These data support that inhibiting Src phosphorylation blocks migration of NSCLC cell spheroids regardless of being cultured on 2D plastic or 3D cellderived matrix. 


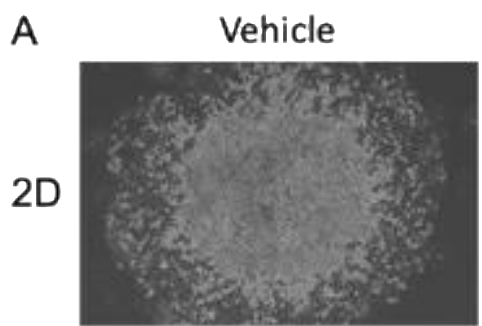

$5 \mu \mathrm{M}$ Saracatinib

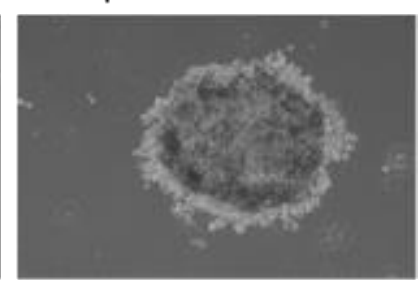

$3 \mathrm{D}$
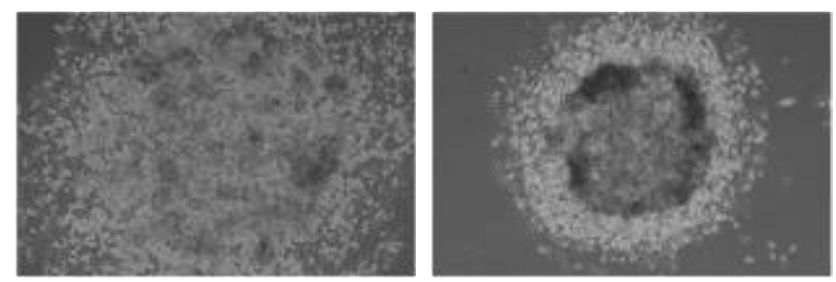

B H2030 Saracatinib Spheroid Assay

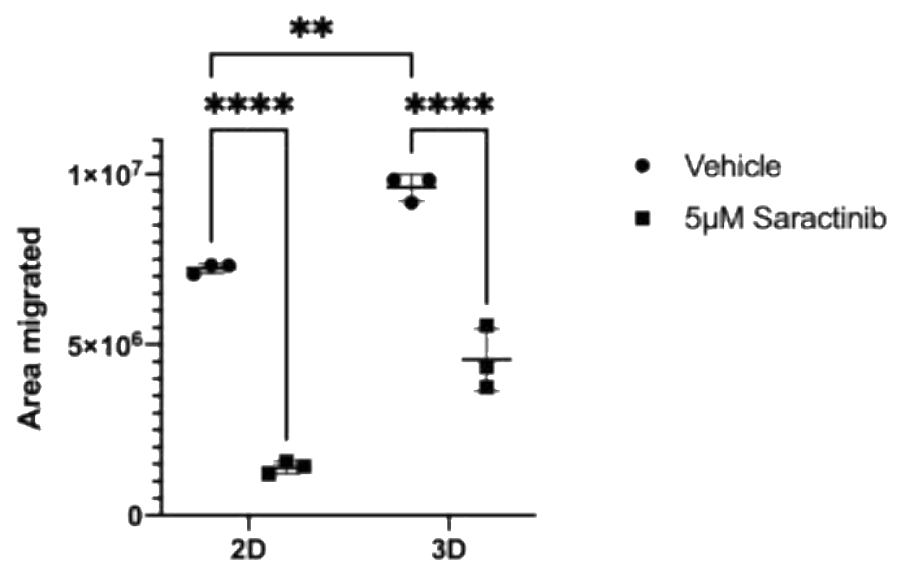

Figure 10. Saracatinib blocks the collective migration of $\mathrm{H} 2030$ lung cancer cells.

H2030 spheroids were grown for four days and transferred to 2D plastic, and 3D IMR90 derived ECM. The spheroids were treated with Saracatinib $(5 \mu \mathrm{M})$ or Vehicle (DMSO). A) Images were captured 48 hours after initial plating. B) Quantification of total migrated area relative to the area of the spheroid. Statistical analysis performed by 2-way ANOVA was performed with multiple comparisons, ${ }^{* *} p<0.01,{ }^{* * * *} p<0.001$; Data presented as mean \pm S.E.M. 


\section{FAK inhibition alters NSCLC cell migration on 2D and 3D.}

FAK phosphorylation is an essential downstream signal for integrin activation. To further investigate how FAK phosphorylation plays a role in cancer cell migration, we treated Hop62, A549, and H2030 lung cancer cells with PF-573228 (Cayman \#S2013), a FAK ${ }^{397}$ autophosphorylation inhibitor.

To determine the concertation of PF-573228 that did not kill Hop62 cells, an Alamar Blue cell viability assay with varying concentrations $(0-20 \mu \mathrm{M})$ of PF573228 at 24, 48, and 72 hours was performed (Figure 11a). Next, Hop62 cells were cultured on $2 \mathrm{D}$ and $3 \mathrm{D}$ with varying concentrations of PF-573228 $(0-5 \mu \mathrm{M})$ to determine which concertation inhibits $F A K^{397}$ phosphorylation (Figure 11b). In Hop62 cells, PF-573228 significantly inhibited FAK ${ }^{397}$ phosphorylation at the 2.5$5 \mu \mathrm{M}$ range on $2 \mathrm{D}$ plastic and $5 \mu \mathrm{M}$ on 3D CDM. 


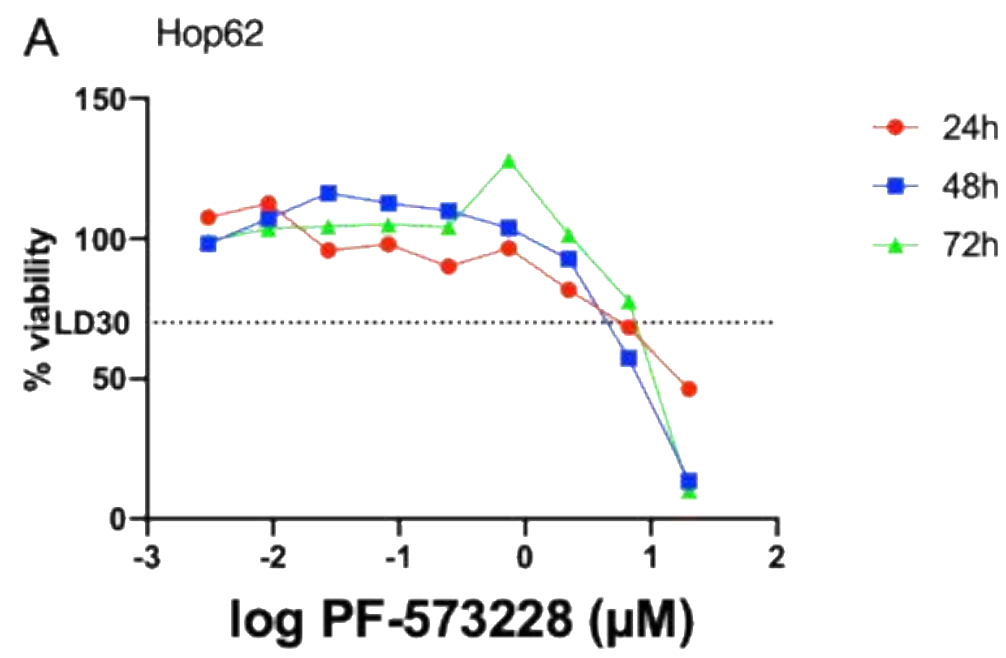

B

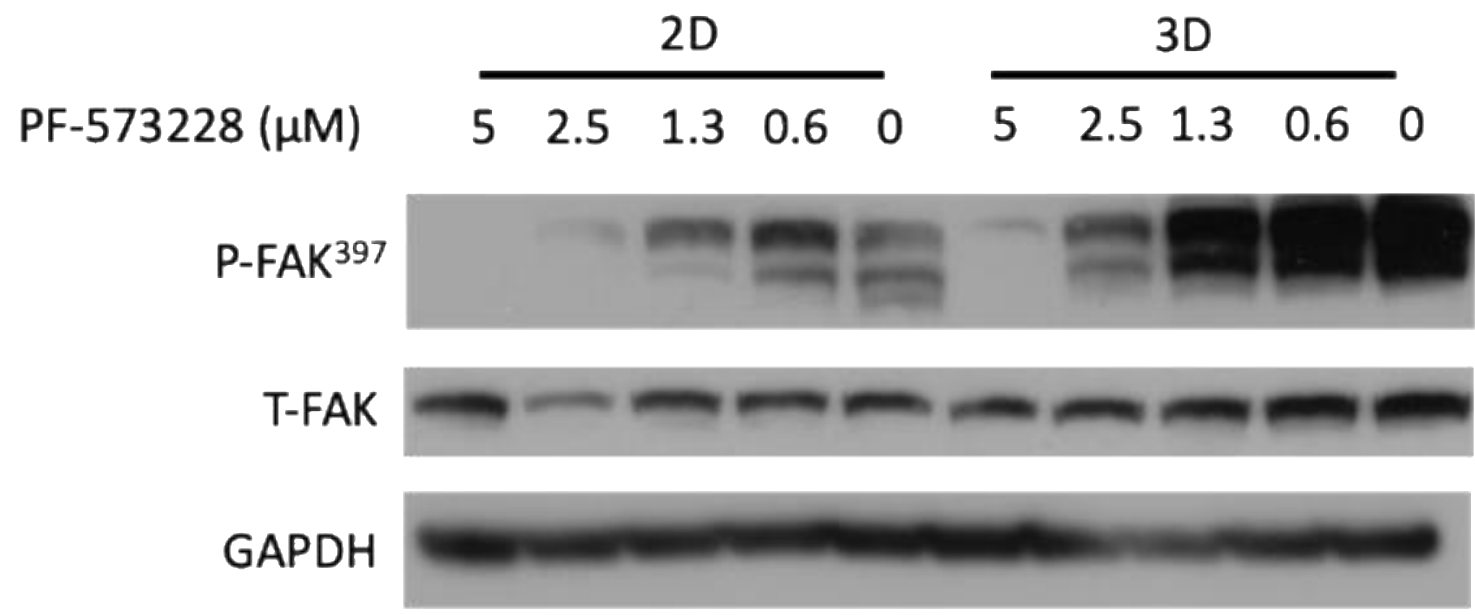

Figure 11. PF-573228 inhibits the autophosphorylation of FAK in Hop62 lung cancer cells.

Hop62 cells were treated with varying concentrations of the FAK inhibitor, PF573228. A) Alamar Blue readings were recorded 24, 48, and 72 hours following treatment. B) Hop62 cells were plated on 2D, and 3D IMR90 derived matrix. The cells were treated with varying concentrations of Saracatinib and collected 72 hours following treatment. Western blot analysis of the extracts was probed for $p$ FAK, FAK, and GAPDH. 
To determine the concertation of PF-573228 that did not kill A549 cells, an Alamar Blue cell viability assay with varying concentrations $(0-20 \mu \mathrm{M})$ of PF573228 at 24, 48, and 72 hours was performed (Figure 12a). Next, A549 cells were cultured on 2D and 3D with varying concentrations of PF-573228 $(0-10 \mu \mathrm{M})$ to determine which concertation inhibits FAK ${ }^{397}$ phosphorylation (Figure 11b). In Hop62 cells, PF-573228 significantly inhibited FAK ${ }^{397}$ phosphorylation at the 5$10 \mu \mathrm{M}$ range on 2D and 3D (Figure12b). 

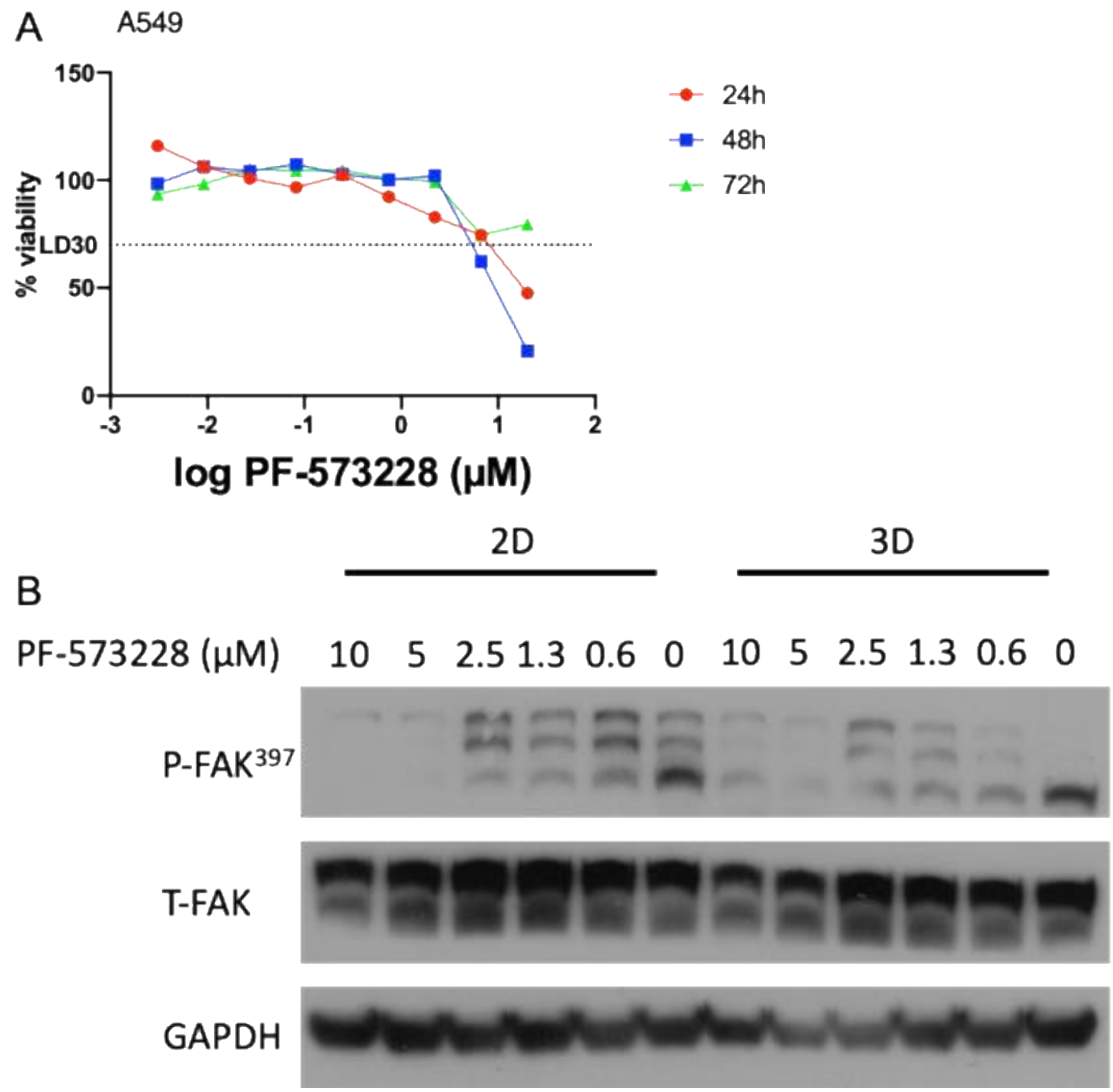

Figure 12. PF-573228 inhibits the autophosphorylation of FAK in A549 lung cancer cells.

A549 cells were treated with varying concentrations of the FAK inhibitor, PF573228. A) Alamar Blue readings were recorded 24, 48, and 72 hours following treatment. B) A549 cells were plated on 2D, and 3D IMR90 derived matrix. The cells were treated with varying concentrations of Saracatinib and collected 72 hours following treatment. Western blot analysis of the extracts was probed for $p$ FAK, FAK, and GAPDH. 
To assess the inhibition of FAK's effect on cancer cell migration, spheroid assays were performed on 2D and 3D with multiple lung cancer cell lines and treated with PF-573228 or not. Hop62 cell spheroids, treated with $5 \mu \mathrm{M}$ of PF-573228 or a DMSO vehicle control, were cultured on 2D plastic and 3D IMR90-derived matrix for 48 hours (Figure 13a). The migrational area of the spheroids was measured and quantified via ImageJ. Hop62 spheroids treated with PF-573228 migrated significantly less on 3D but not 2D compared to the vehicle control (Figure 13b). 
A

Vehicle

$5 \mu \mathrm{M}$ PF-573228

$2 \mathrm{D}$
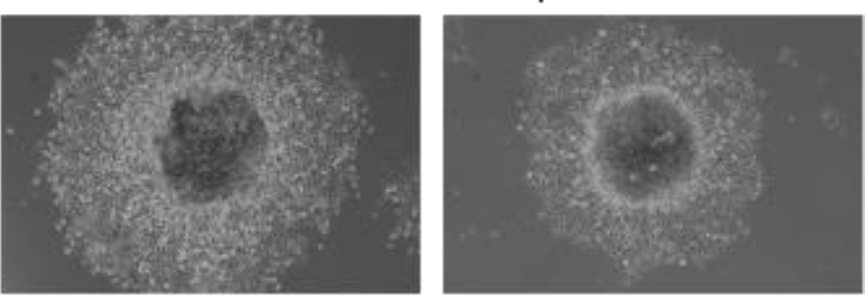

3D
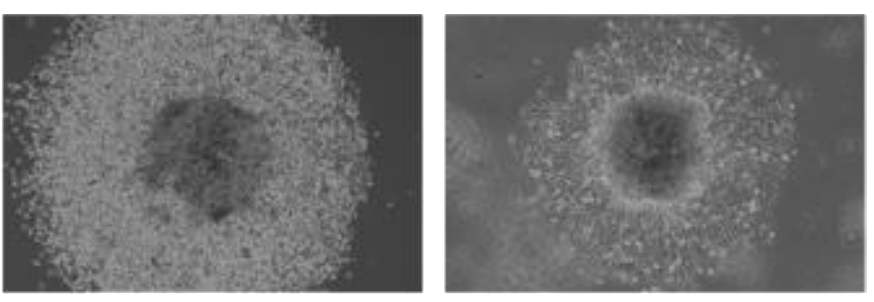

B Hop62 FAKi Spheroid Assay

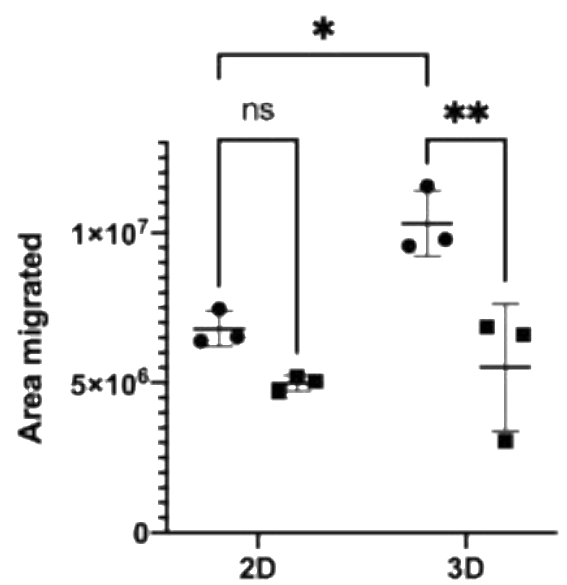

- Vehicle

- $5 \mu \mathrm{M}$ PF -573228

Figure 13. PF-573228 decreases the collective cell migration of Hop62 lung cancer cells.

Hop62 spheroids were grown for four days and transferred to 2D plastic, and 3D IMR90 derived ECM. The spheroids were treated with PF-573228 $(5 \mu \mathrm{M})$ or Vehicle (DMSO). A) Images were captured 48 hours after initial plating. B) Quantification of total migrated area relative to the area of the spheroid. Statistical analysis performed by 2-way ANOVA was performed with multiple comparisons, ${ }^{*} p<0.05,{ }^{* *} p<0.01$; Data presented as mean \pm S.E.M. 
A549 cell spheroids, treated with $5 \mu \mathrm{M}$ of PF-573228 or a DMSO vehicle control, were cultured on 2D plastic and 3D IMR90-derived matrix for 48 hours (Figure 14a). The migrational area of the spheroid was measured and quantified via ImageJ. There was no statistically significant difference in the migration of A549 spheroids treated with PF-573228 on 2D or 3D (Figure 14b).

These results suggest inhibition of $\mathrm{FAK}^{397}$ phosphorylation's influence on cell migration is cell condition (2Dv3D) and cell line (Hop62vA549vH2030) dependent. 
A
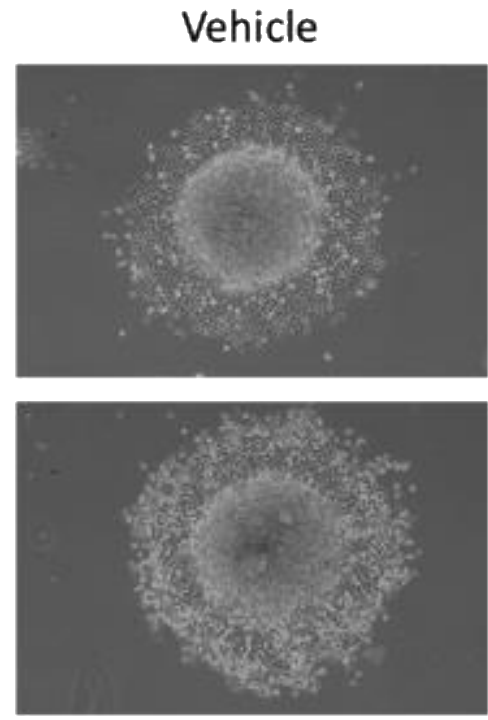

$5 \mu \mathrm{M}$ PF-573228
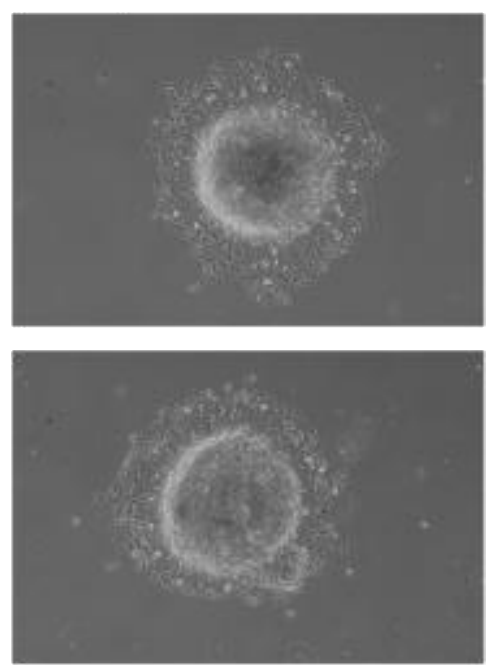

B A549 FAKi Spheroid Assay

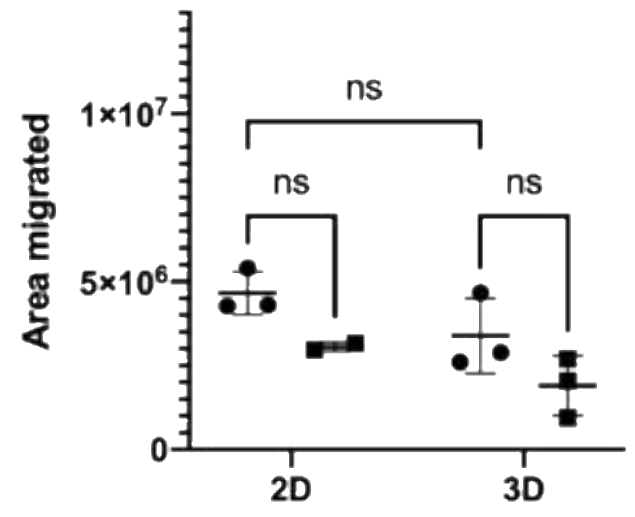

- Vehicle

- $5 \mu \mathrm{M}$ PF-573228

Figure 14. PF-573228 does not impact the collective cell migration of A549 lung cancer cells.

A549 spheroids were grown for four days and transferred to 2D plastic, and 3D IMR90 derived ECM. The spheroids were treated with PF-573228 $(5 \mu \mathrm{M})$ or Vehicle (DMSO). A) Images were captured 48 hours after initial plating. B) Quantification of total migrated area relative to the area of the spheroid. Statistical analysis performed by 2-way ANOVA was performed with multiple comparisons; Data presented as mean \pm S.E.M. 


\section{Co-cultured fibroblast migration is Src-independent on 3D.}

The tumor microenvironment is composed of many different cell types. Of those cell types, fibroblasts are the most important for ECM production and degradation. To investigate how fibroblasts play a role in cancer cell migration, spheroid assays were performed, culturing both cancer cells and fibroblasts together. In a 1:5 Hop62: IMR90GFP ratio, co-cultured spheroids were created, transferred to 2D and 3D, and cultured for 72 hours (Figure 15a). Light and fluorescence microscopy photos were captured, then measured and quantified using ImageJ. The cancer cells were not fluorescently labeled, but the fibroblasts were, allowing for separate quantification between the cancer cell and fibroblast migration. The area cancer cell migration was not significantly changed on 2D or 3D, but the area of fibroblast migration was significantly increased on 3D compared to 2D (Figure 15b). 

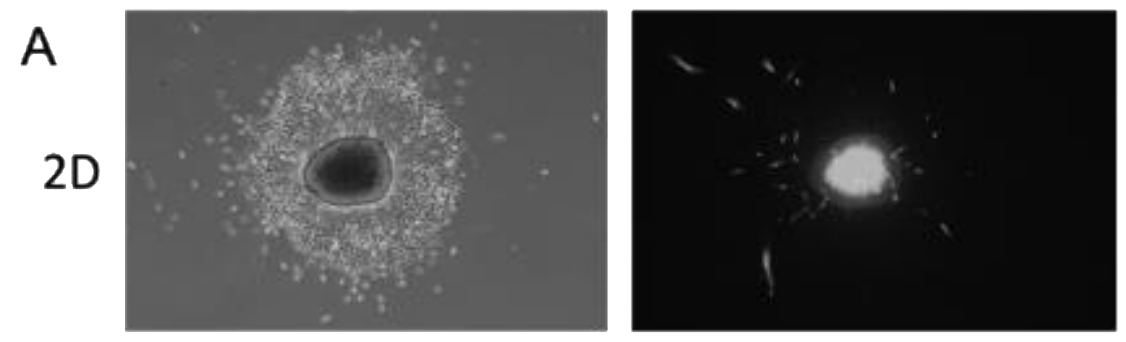

$3 \mathrm{D}$
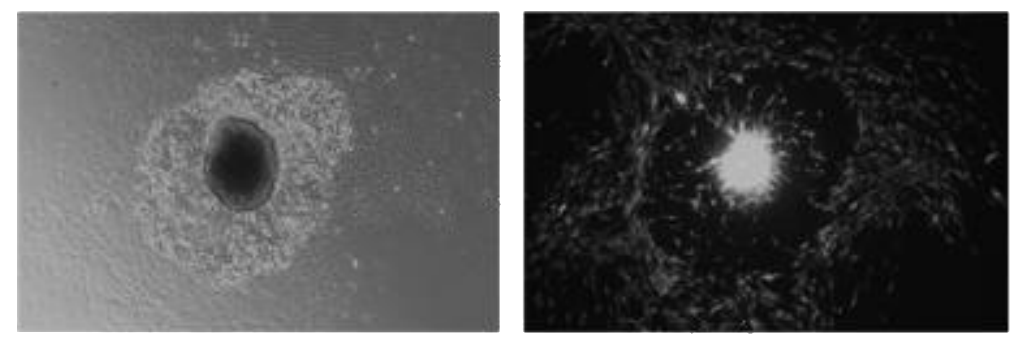

B

\section{A549 Cancer Cell and Fibroblast Mlgration}

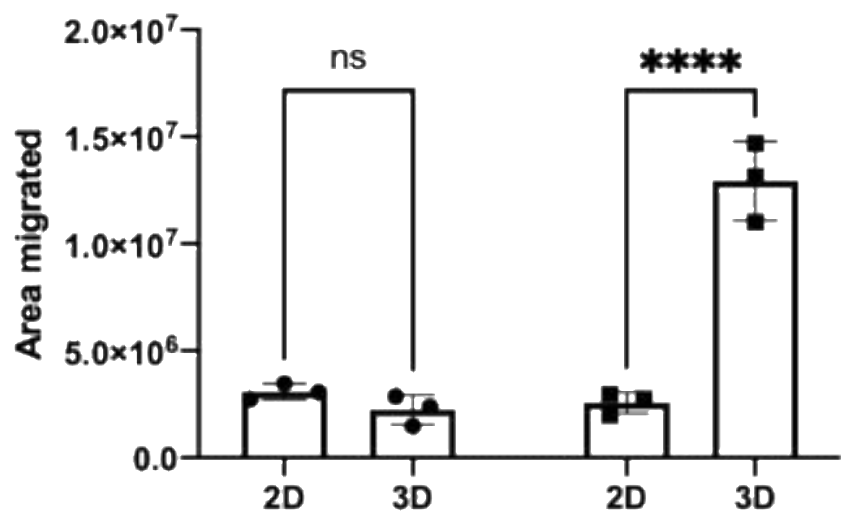

- Cancer Cells

- Fibroblasts

Figure 15. IMR90 lung fibroblasts co-cultured with A549 lung cancer cells migrate more on 3D CDM.

Co-cultured spheroids consisting of A549 and GFP labeled IMR90 lung fibroblasts in a 1:5 ratio, respectively, were grown for 4 days and transferred to 2D plastic or 3D IMR90 derived ECM. A) Pictures were taken 72 hours following transfer (bright field on the left, fluorescence on the right). B) Quantification of total migrated area relative to the area of spheroid Statistical analysis performed by 2-way ANOVA was performed with multiple comparisons, ${ }^{* *} p<0.01$, ${ }^{* * * *}$ $p<0.001$; Data presented as mean \pm S.E.M. 
We previously showed that treatment of an Src inhibitor, Saracatinib, significantly reduced the migration of Hop62, A549, and H2030 cells on 2D and 3D. Because we saw such a noticeable migrational and phenotypic difference in the migration of co-cultured fibroblasts on 2D compared to $3 \mathrm{D}$, we wanted to investigate the impact of Src inhibition on our co-cultured system. First, we needed to determine the concertation of Saracatinib that was not lethal to IMR90 cells. To do this, an Alamar Blue cell viability assay with varying concentrations $(0-20 \mu \mathrm{M})$ of Saracatinib at 24, 48, and 72 hours was performed (Figure 16a). Next, IMR90 cells were cultured on 2D and 3D with varying concentrations of Saracatinib (0$10 \mu \mathrm{M})$ to determine which concertation inhibits Src phosphorylation (Figure 23b). Saracatinib significantly inhibited Src phosphorylation from the $0.6-10 \mu \mathrm{M}$ range in IMR90 cells on both 2D and 3D (Figure 16b). 

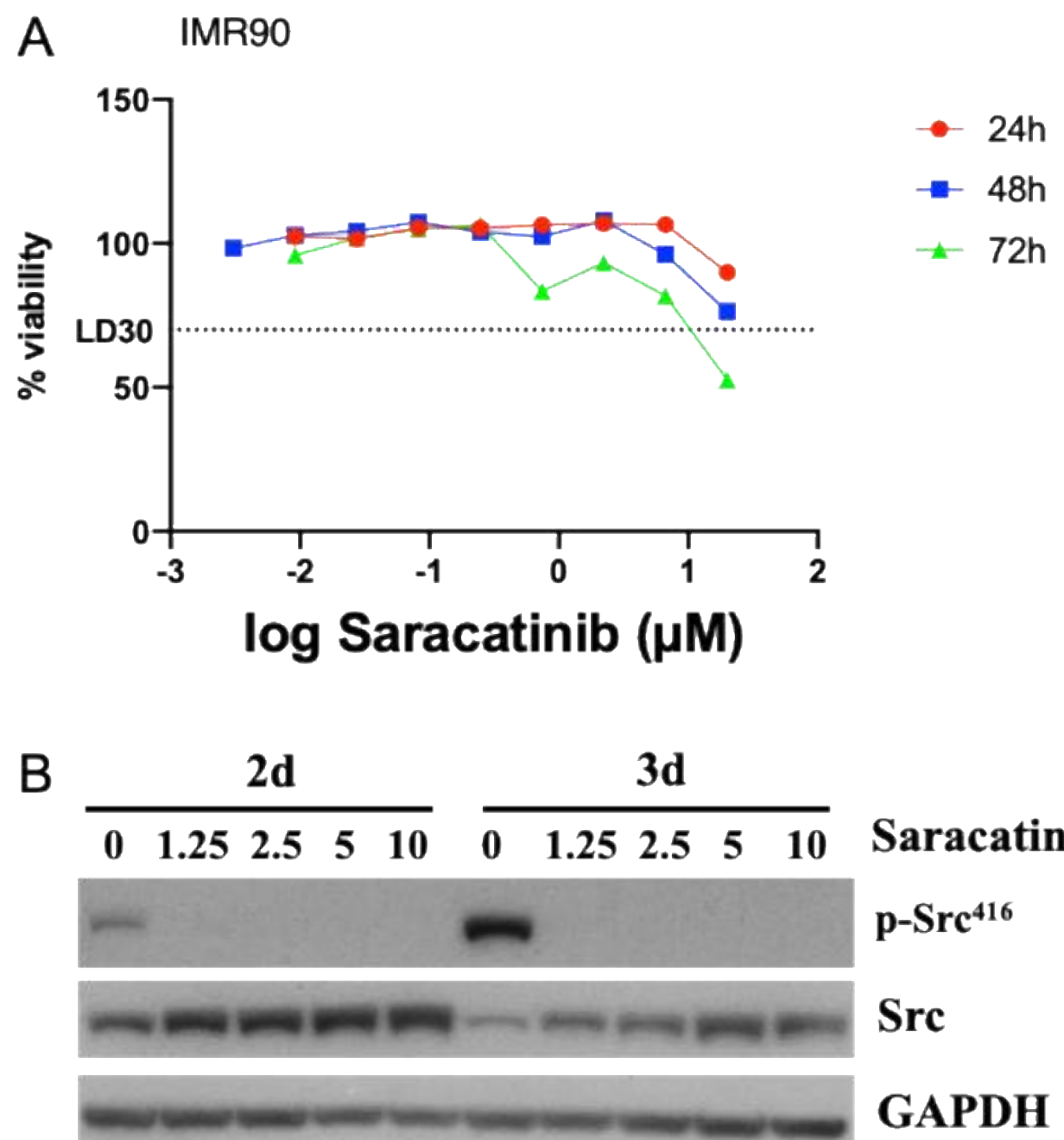

Figure 16. Saracatinib inhibits Src phosphorylation in IMR90 lung fibroblasts.

IMR90 lung fibroblasts were treated with varying concentrations of Saracatinib.

A) Alamar Blue readings were recorded 24, 48, and 72 hours following treatment. B) IMR90 lung fibroblasts were plated on 2D, and 3D IMR90 derived matrix. The cells were treated with varying concentrations of Saracatinib and collected 72 hours following treatment. Western blot analysis of the extracts was probed for $p$ src, src, and GAPDH. 
To investigate how Src inhibition impacted the cancer cell and fibroblast migration, a spheroid co-culture assay was performed. In a 1:5 A549: IMR90GFP ratio, co-cultured spheroids, treated with $5 \mu \mathrm{M}$ of Saracatinib or DMSO vehicle, were cultured on 2D and 3D for 72 hours (Figure 17a). Light and fluorescence microscopy photos were captured, then measured and quantified using ImageJ. The cancer cells were not fluorescently labeled, but the fibroblasts were, allowing for separate quantification between the cancer cell and fibroblast migration (Figure 17b). When treated with saracatinib, cancer cell and fibroblast migration decreased on 2D and 3D. Untreated spheroid cancer cell migration did not differ on $2 \mathrm{D}$ and $3 \mathrm{D}$. However, fibroblast migration significantly increased on 3D compared to 2D. This data suggests fibroblasts migrate more on 3D and in an Src-dependent manner on 2D, but not 3D, when co-cultured with A549 cancer cells. 


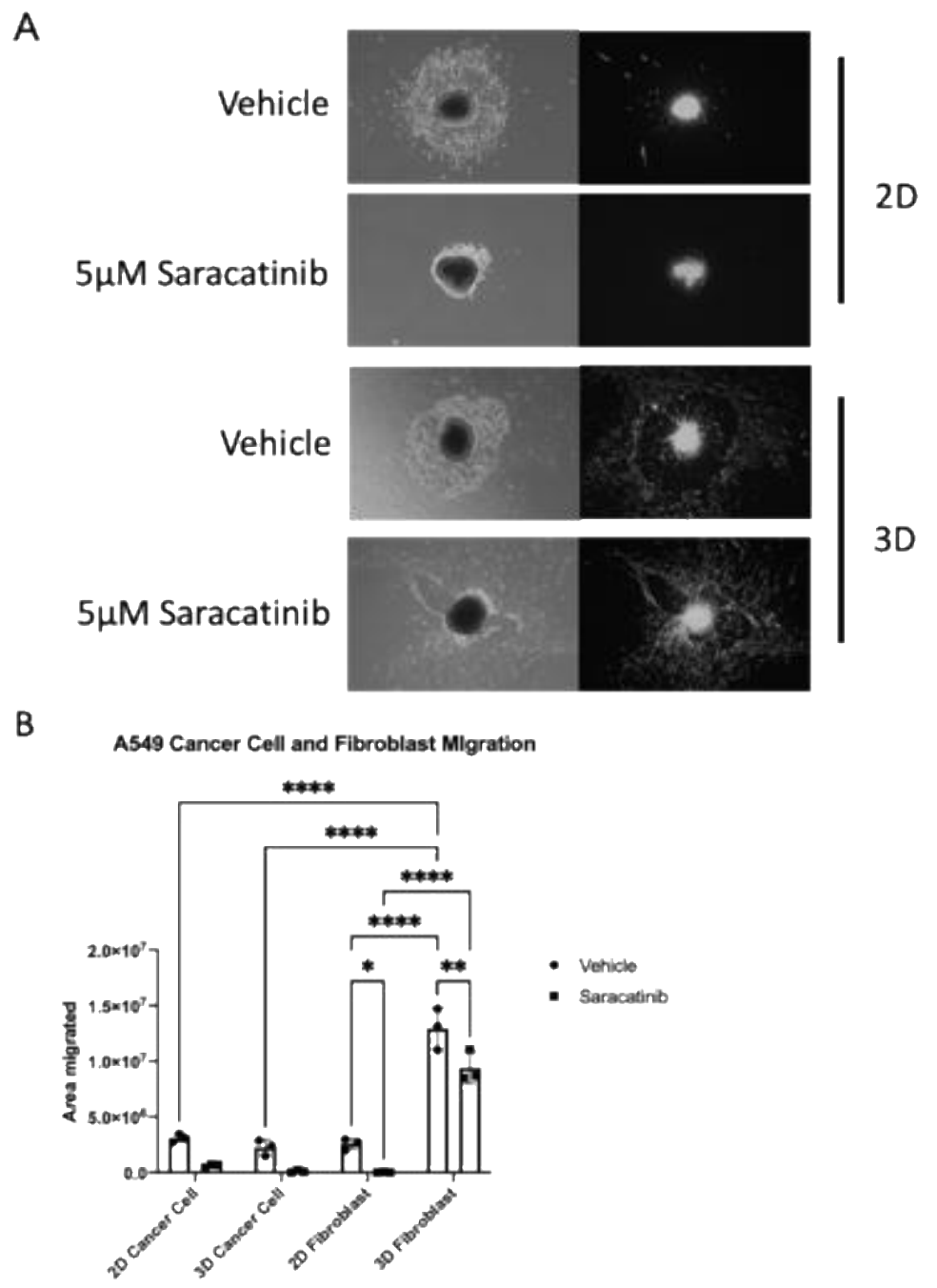

Figure 17. Saracatinib blocks the migration of IMR90 lung fibroblasts cocultured with A549 lung cancer cells on 2D but not 3D.

Co-cultured spheroids consisting of A549 and IMR90 lung fibroblasts in a 1:5 ratio, respectively, were grown for 4 days and transferred to $2 \mathrm{D}$ plastic or $3 \mathrm{D}$ IMR90 derived ECM. The spheroids were treated with Saracatinib $(5 \mu \mathrm{M})$ or Vehicle (DMSO). A) Pictures were taken 72 hours following transfer (bright field on the left, fluorescence on the right). B) Quantification of total migrated area relative to the area of the spheroid. Statistical analysis performed by 2-way ANOVA was performed with multiple comparisons, ${ }^{* *} p<0.01$, ${ }^{\star * \star *} p<0.001$; Data presented as mean \pm S.E.M. 
In a 1:5 Hop62: IMR90GFP ratio, co-cultured spheroids, treated with $5 \mu \mathrm{M}$ of Saracatinib, or DMSO vehicle, was cultured on 2D and 3D for 72 hours (Figure 18a). Light and fluorescence microscopy photos were captured, then measured and quantified using ImageJ. The cancer cells were not fluorescently labeled, but the fibroblasts were, allowing for separate quantification between the cancer cell and fibroblast migration (Figure 18b). When treated with saracatinib, cancer cell migration was significantly decreased on 2D and appeared to decrease on 3D. However, fibroblast migration significantly decreased on 3D but not 2D. Untreated spheroid cancer cell migration did not differ on 2D and 3D. However, fibroblast migration significantly increased on 3D compared to 2D. This data suggests fibroblasts migrate more on 3D and in an Src-dependent manner on 2D when co-cultured with Hop62 cancer cells. 
A


B Hop62 Cancer Cell and Fibroblast Mlgration

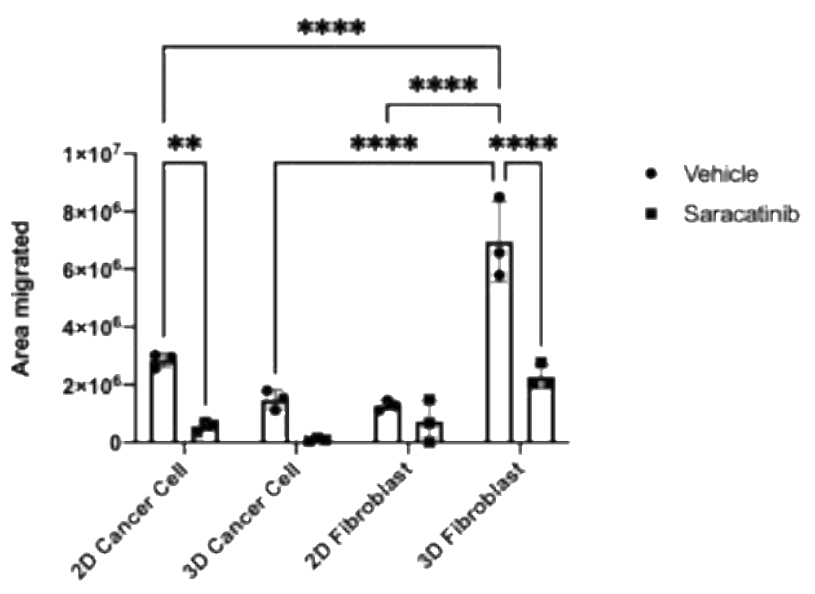

Figure 18. Saracatinib blocks the migration of IMR90 lung fibroblasts cocultured with Hop62 lung cancer cells on 2D but not 3D.

Co-cultured spheroids consisting of Hop62 and IMR90 lung fibroblasts in a 1:5 ration, respectively, were grown for 4 days and transferred to 2D plastic or 3D IMR90 derived ECM. The spheroids were treated with Saracatinib $(5 \mu \mathrm{M})$ or Vehicle (DMSO). A) Pictures were taken 72 hours following transfer (bright field on the left, fluorescence on the right). B) Quantification of total migrated area relative to the area of the spheroid. Statistical analysis performed by 2-way ANOVA was performed with multiple comparisons, ${ }^{* *} p<0.01,{ }^{* * *} p<0.001$; Data presented as mean \pm S.E.M. 
To investigate fibroblast migration independently of cancer cells on 2D, IMR90 fibroblasts were cultured at a low density on 2D and treated with Saracatinib or not (Figure 19a and b). Treatment with Saracatinib significantly decreased fibroblast displacement, acceleration, and speed on 2D (Figure 19c). 
A
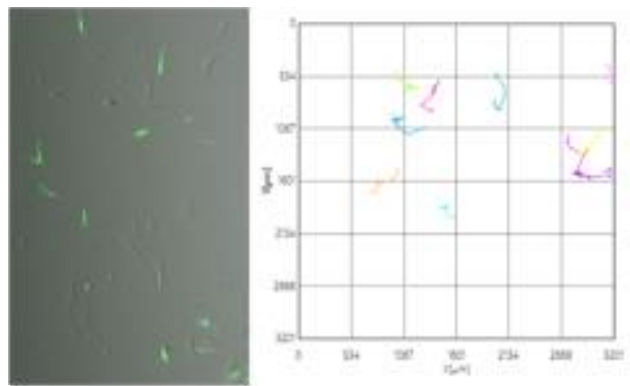

B
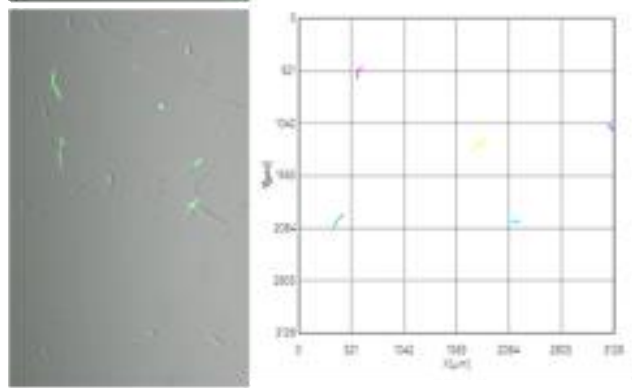

C

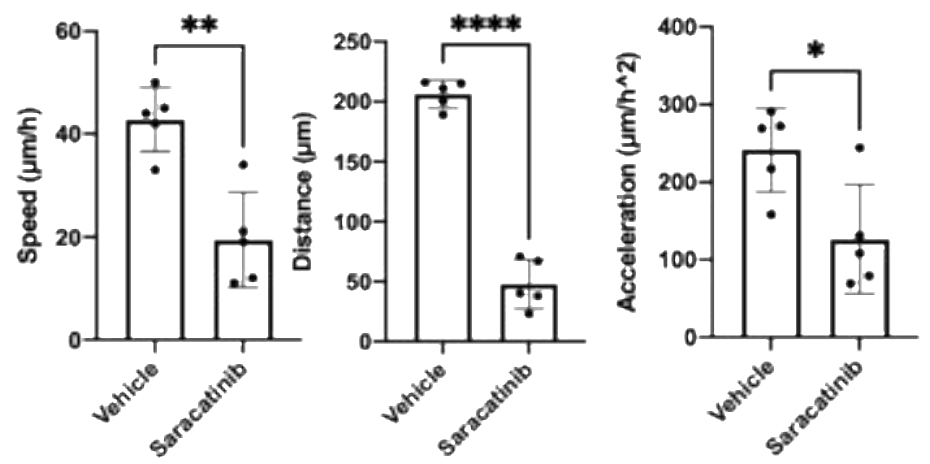

Figure 19. Saracatinib impacts the single cell migration of IMR90 lung fibroblasts on 2D.

IMR90 cells were plated at a low concentration on 2D plastic. Time-lapse imaging was recorded, imaging every 15 minutes for 24 hours. IMR90s were treated with either A) Vehicle (DMSO) or B) Saracatinib $(5 \mu M)$ C) Single-cell speed, distance, and acceleration were recorded. Statistical analysis performed by 2-way ANOVA was performed with multiple comparisons. Statistical analysis performed by 2-way ANOVA was performed with multiple comparisons, * $p<0.05^{\star *} p<0.01,{ }^{* * *} p<0.001$; Data presented as mean \pm S.E.M. 
To investigate fibroblast migration independently of cancer cells on 3D, IMR90 fibroblasts were cultured at a low density on 3D and treated with Saracatinib or not (Figure 20a and b). Treatment with Saracatinib did not significantly decrease fibroblast displacement, acceleration, or speed on 3D (Figure 20c). 
A
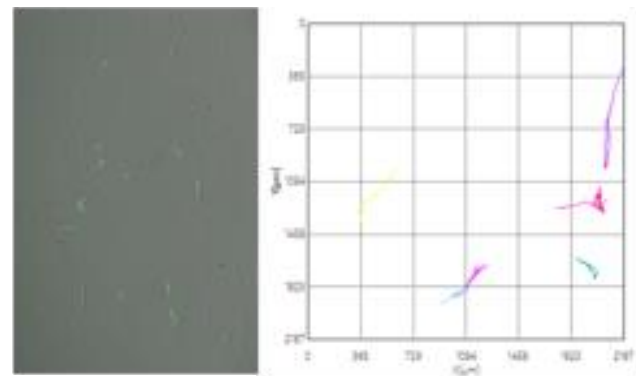

B
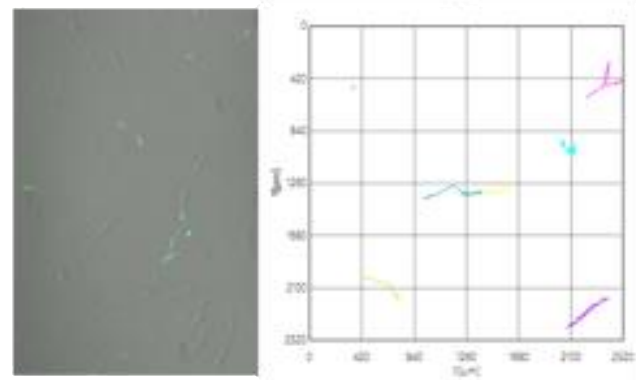

C

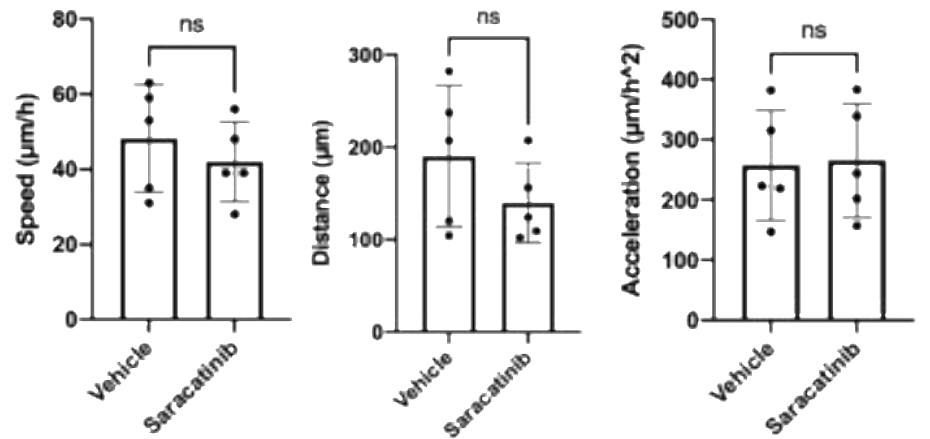

Figure 20. Saracatinib does not impact the single cell migration of IMR90 lung fibroblasts on 3D.

IMR90 cells were plated at a low concentration on 3D IMR90 derived ECM. Time-lapse imaging was recorded, imaging every 15 minutes for 24 hours. IMR90s were treated with either A) Vehicle (DMSO) or B) Saracatinib $(5 \mu \mathrm{M})$ C) Single-cell speed, distance, and acceleration were recorded. Statistical analysis performed by 2-way ANOVA was performed with multiple comparisons. 


\section{DISCUSSION}

In this study, we investigated the role of integrin signaling, specifically Src, in the context of NSCLC cell and fetal lung fibroblast migration on fetal lung fibroblast-derived extracellular matrix. Our lab has previously demonstrated that lung cancer cells alter their migration and integrin signaling on 3D cell-derived matrix compared to 2D plastic [30]. Historically, cancer cell migration has been studied in vitro utilizing plastic dishes. Here, we use a system using cell-derived matric as a more physiologically relevant cell culture method and study the interaction between cancer cells and the extracellular matrix (ECM). Fibroblasts are the primary ECM producers and remodelers. This study highlighted the differential cancer cell and fibroblast migration on 2D and 3D while perturbing integrin signaling.

Schertzer et al. demonstrated that the single cell migration of cancer cells is differentially altered on $2 \mathrm{D}$ and $3 \mathrm{D}$. Furthermore, Src and FAK phosphorylation was increased in NSCLC cells cultured on 3D. To further investigate this phenotype, we developed a collective cell spheroid migration assay on 2D plastic and 3D CDM. In multiple cell lines, collective cell migration was increased on 3D CDM (Figures 1 and 2), suggesting that the ECM influenced cancer cell migration. To increase scientific rigor, a scratch wound assay on 2D and 3D 
CDM was performed using A549 NSCLC cells (Figure 3), where collective cell migration increased on 3D CDM. This data suggested that culturing NSCLC cells on 3D CDM influenced collective cancer cell migration.

As previously stated, our group has shown that Src and FAK signaling is increased in NSCLC cells cultured on 3D CDM. Src and FAK's role in cell adhesion and migration is well documented [17]. Saracatinib is a welldocumented Src inhibitor [36], as well as a known inhibitor of EGFR and Abl, and has been tested in multiple clinical trials but failed due to lack and efficacy and harsh side effects. Regardless of the failed trials, Saracatinib is a potent Src inhibitor. To investigate how inhibiting Src impacts NSCLC collective cell migration, spheroid assays were performed with multiple NSCLC cell lines on 2D and 3D treating with $5 \mu \mathrm{M}$ Saracatinib or DMSO vehicle (Figures 8-10). Inhibition of Src completely blocked collective cell migration regardless of cell culture conditions or cell line. PF-573228 inhibits the phosphorylation of FAK at its 397 autophosphorylation site. To investigate how inhibiting $\mathrm{FAK}^{397}$ phosphorylation impacts NSCLC collective cell migration, spheroid assays were performed with Hop62 and A549 NSCLC cell lines on 2D and 3D, and treated with 5 MM PF573228 or DMSO vehicle (Figures 8-9). Interestingly enough, Hop62 collective cell migration was decreased by PF-573228 on 3D, but not 2D. However, A549 collective cell migration was not affected by PF-573228 treatment regardless of the condition. This data suggested that Src plays a more critical role in cancer cell migration than FAK. 
Fibroblasts are among the most abundant cell types in the tumor microenvironment and are responsible for remodeling and producing ECM. We developed a system to co-culture cancer cells and fibroblasts together to create co-cultured spheroids. When monitoring collective cell migration of A549-IMR90 co-cultured spheroids, IMR90 fibroblast migration was significantly increased on 3D compared to 2D. This data suggests that when IMR90 cells interact with the cell-derived ECM, migrational phenotypes are enhanced. Because inhibiting Src completely blocked the collective cell migration of cancer cells, we wanted to investigate co-cultured spheroid migration when Src is inhibited with Saracatinib. Interestingly enough, Saracatinib decreased cancer cell migration regardless of conditions, where fibroblast migration was also decreased, but not to the same extent. Compared to the cancer cells, the fibroblasts migrated significantly more even when treated with Saracatinib. This data suggested that fibroblasts migrated in a more Src-independent manner than cancer cells on 3D CDM.

Because we saw a difference in the cancer cell and fibroblast migration when treated with Saracatinib, we investigated the migration of fibroblasts on 2D and 3D, treated with Saracatinib or DMSO vehicle, independently of cancer cells via single cell tracking. When treated with Saracatinib, fibroblast speed, acceleration, and distance were significantly different between the vehicle-treated group on 2D, but there was no difference between groups on 3D. This result further supported our hypothesis that fibroblasts migrated in an Src-independent manner on 3D CDM. 
It is well understood that fibroblasts play a critical role in facilitating cancer cell metastasis [37]. This study is crucial in understanding how fibroblasts migrate in the presence of cancer cells and not. Our results suggest that cancer cells cultured on 3D cell-derived matrix migrate in a Src-dependent manner while fibroblasts do not. These findings are important because they may explain why Src inhibitors, such as Saracatinib, may have failed clinical trials due to effectiveness. It is also important to note that we see the differences in modes of migration of fibroblasts on 2D compared to $3 \mathrm{D}$ cell-derived matrix, a model proposed to be more physiologically relevant. Understanding the mechanisms of migration of cancer cells and fibroblasts when interacting with the ECM may be crucial in targeting cancer metastasis. 


\section{SUMMARY}

Lung cancer is the most lethal type of cancer in males and females primarily due to its metastatic spread. There has yet to be FDA-approved treatment to treat lung cancer metastasis. To approach this knowledge gap, we investigated the interactions between multiple components of the tumor microenvironment. To this extent, we established a cell culture system that mimics physiological conditions. Briefly, fibroblasts are cultured at confluence, decellularized on the dish, and what's left behind is a 3D fibroblast-deposited matrix. Utilizing this system allows us to study the interaction between NSCLC cells and fibroblast-derived ECM.

When NSCLC cells are cultured on a fibroblast-derived matrix, their velocity, directionality, and displacement increase. To investigate cancer cell migration, we performed spheroid and scratch wound assays. Our results revealed that cancer cell migration increased on 3D in multiple cell lines and multiple migration assays. Because SRC signaling increased in cancer cells cultured on 3D, we pharmacologically inhibited SRC in our spheroid assay. Inhibiting SRC significantly decreased migration regardless of culture conditions. To further investigate how fibroblasts impact cancer cell migration, co-cultured spheroid assays were performed. Fibroblast migration increased on 3D, but cancer cell migration did not. We treated the co-cultured spheroids with an SRC 
inhibitor. To our surprise, fibroblast migration decreased on 2D but not 3D. These studies demonstrate that cancer cells and fibroblasts migrated through different mechanisms on 3D fibroblast-derived matrix. Future studies will investigate the specific mechanisms by which cancer cells and fibroblasts migrate on 3D fibroblast-derived matrix. 


\section{REFERENCES}

1. Key Statistics for Lung Cancer. 2019.

2. Brody, H., Lung cancer. Nature, 2014. 513(7517): p. S1.

3. Anderson, R.L., et al., A framework for the development of effective antimetastatic agents. Nat Rev Clin Oncol, 2019. 16(3): p. 185-204.

4. Chiang, A.C., and J. Massague, Molecular basis of metastasis. N Engl J Med, 2008. 359(26): p. 2814-23.

5. Snijders, R.J., et al., First-trimester trisomy screening: nuchal trans/ucency measurement training and quality assurance to correct and unify technique. Ultrasound Obstet Gynecol, 2002. 19(4): p. 353-9.

6. Denton, A.E., E.W. Roberts, and D.T. Fearon, Stromal Cells in the Tumor Microenvironment. Adv Exp Med Biol, 2018. 1060: p. 99-114.

7. Chen, $X$. and E. Song, Turning foes to friends: targeting cancerassociated fibroblasts. Nat Rev Drug Discov, 2019. 18(2): p. 99-115.

8. Shen, M. and Y. Kang, Complex interplay between tumor microenvironment and cancer therapy. Front Med, 2018. 12(4): p. 426439.

9. Walker, C., E. Mojares, and A. Del Rio Hernandez, Role of Extracellular Matrix in Development and Cancer Progression. Int J Mol Sci, 2018. 19(10).

10. Ricard-Blum, S., The collagen family. Cold Spring Harb Perspect Biol, 2011. 3(1): p. a004978.

11. lozzo, R.V. and L. Schaefer, Proteoglycan form and function: $A$ comprehensive nomenclature of proteoglycans. Matrix Biol, 2015. 42: $\mathrm{p}$. 11-55.

12. Altorki, N.K., et al., The lung microenvironment: an important regulator of tumour growth and metastasis. Nat Rev Cancer, 2019. 19(1): p. 9-31.

13. Hanahan, D. and L.M. Coussens, Accessories to the crime: functions of cells recruited to the tumor microenvironment. Cancer Cell, 2012. 21(3): $p$. 309-22.

14. Van Doren, S.R., Matrix metalloproteinase interactions with collagen and elastin. Matrix Biol, 2015. 44-46: p. 224-31.

15. Takada, Y., X. Ye, and S. Simon, The integrins. Genome Biol, 2007. 8(5): p. 215.

16. Cooper, J. and F.G. Giancotti, Integrin Signaling in Cancer: Mechanotransduction, Stemness, Epithelial Plasticity, and Therapeutic Resistance. Cancer Cell, 2019. 35(3): p. 347-367. 
17. Mitra, S.K. and D.D. Schlaepfer, Integrin-regulated FAK-Src signaling in normal and cancer cells. Curr Opin Cell Biol, 2006. 18(5): p. 516-23.

18. Colo, G.P., E.M. Lafuente, and J. Teixido, The MRL proteins: adapting cell adhesion, migration and growth. Eur J Cell Biol, 2012. 91(11-12): p. 861-8.

19. Dongre, A. and R.A. Weinberg, New insights into the mechanisms of epithelial-mesenchymal transition and implications for cancer. Nat Rev Mol Cell Biol, 2019. 20(2): p. 69-84.

20. Mittal, V., Epithelial Mesenchymal Transition in Tumor Metastasis. Annu Rev Pathol, 2018. 13: p. 395-412.

21. Liao, T.T. and M.H. Yang, Revisiting epithelial-mesenchymal transition in cancer metastasis: the connection between epithelial plasticity and stemness. Mol Oncol, 2017. 11(7): p. 792-804.

22. Vinci, M., C. Box, and S.A. Eccles, Three-dimensional (3D) tumor spheroid invasion assay. J Vis Exp, 2015(99): p. e52686.

23. Vang Mouritzen, M. and H. Jenssen, Optimized Scratch Assay for In Vitro Testing of Cell Migration with an Automated Optical Camera. J Vis Exp, 2018(138).

24. Rommerswinkel, N., et al., Analysis of cell migration within a threedimensional collagen matrix. J Vis Exp, 2014(92): p. e51963.

25. Chen, H.C., Boyden chamber assay. Methods Mol Biol, 2005. 294: p. 1522.

26. Kleinman, H.K. and G.R. Martin, Matrigel: basement membrane matrix with biological activity. Semin Cancer Biol, 2005. 15(5): p. 378-86.

27. Benton, G., et al., Multiple uses of basement membrane-like matrix (BME/Matrigel) in vitro and in vivo with cancer cells. Int J Cancer, 2011. 128(8): p. 1751-7.

28. Saforo, D., et al., Primary lung cancer samples cultured under microenvironment-mimetic conditions enrich for mesenchymal stem-like cells that promote metastasis. Sci Rep, 2019. 9(1): p. 4177.

29. Kleinman, H.K. and K. Jacob, Invasion assays. Curr Protoc Cell Biol, 2001. Chapter 12: p. Unit 122.

30. Scherzer, M.T., et al., Fibroblast-Derived Extracellular Matrices: An Alternative Cell Culture System That Increases Metastatic Cellular Properties. PLoS One, 2015. 10(9): p. e0138065.

31. Soucy, P.A. and L.H. Romer, Endothelial cell adhesion, signaling, and morphogenesis in fibroblast-derived matrix. Matrix Biol, 2009. 28(5): p. 273-83.

32. Nazemi, M. and E. Rainero, Cross-Talk Between the Tumor Microenvironment, Extracellular Matrix, and Cell Metabolism in Cancer. Front Oncol, 2020. 10: p. 239.

33. Alexander, J. and E. Cukierman, Cancer associated fibroblast: Mediators of tumorigenesis. Matrix Biol, 2020. 91-92: p. 19-34.

34. Guarino, M., Src signaling in cancer invasion. J Cell Physiol, 2010. 223(1): p. 14-26. 
35. Caner, A., E. Asik, and B. Ozpolat, SRC Signaling in Cancer and Tumor Microenvironment. Adv Exp Med Biol, 2021. 1270: p. 57-71.

36. Giaccone, G. and P.A. Zucali, Src as a potential therapeutic target in nonsmall-cell lung cancer. Ann Oncol, 2008. 19(7): p. 1219-1223.

37. Ishii, G., A. Ochiai, and S. Neri, Phenotypic and functional heterogeneity of cancer-associated fibroblast within the tumor microenvironment. Adv Drug Deliv Rev, 2016. 99(Pt B): p. 186-196. 


\title{
CURRICULUM VITAE
}

\author{
Austin M. Krueger \\ University of Louisville School of Medicine \\ Department of Pharmacology and Toxicology \\ 505 S. Hancock St. (CTRB 252G) \\ Louisville, KY, 40202 \\ (484) $-343-5958$
}

\section{Education:}

2010 - 2014 Saint Xavier High School (GPA 3.9), Louisville, KY

2014 - 2018 BS in Cellular Biology and Physiology (GPA 3.34), University of Louisville, Department of Biology, Louisville, KY

\section{Honors and Awards:}

$2014 \quad$ Scholarship, Kosair Charities

2014 - 2018 Scholarship, University of Louisville Board of Trustees

2017 - 2018 Dean's List, University of Louisville

\section{Research Experience:}

2015-2018 Undergraduate Research Assistant, Dr. Mark Running, Department of Biology, University of Louisville, Louisville, KY

2017

- Expression of Lignolytic Enzymes from White-rot Fungi Undergraduate Research Assistant, Dr. Juliane Arteel, R25 Cancer Education Program, Department of Pharmacology and Toxicology, University of Louisville, Louisville, KY -

2018 - present Rapamycin Blunts Vinyl Chloride (VC)-induced liver injury Graduate student, Dr. Leah Siskind, Department of Pharmacology and Toxicology, University of Louisville, Louisville, KY - The role of Src in NSCLC cell and lung fibroblast migration on $3 D$ cell-derived matrix

\section{Teaching Experience:}

2019 Antibiotics and Antifungals, Dental Hygiene, University of, Louisville, Louisville, KY

2020 Antibiotics and Antifungals, Dental Hygiene, University of Louisville, Louisville, KY

\section{Oral Presentations:}


Expression of Lignolytic Enzymes from White-rot Fungi, May 2017, Department of Biology Symposium

Rapamycin Blunts Vinyl Chloride (VC)-induced liver injury, August 2017, Louisville Summer Research Symposium

Cancer Associated Mesenchymal Cells Influence the Metastatic Signaling of Cancer Cells: The predominant ECM-producing cells are fibroblasts, September 2019, Research Louisville Research Symposium

Cancer Associated Mesenchymal Cells Influence the Metastatic Signaling of Cancer Cells: The predominant ECM-producing cells are fibroblasts, September 2019, CSHL: Mechanisms of Metastasis Symposium

\section{Research Publications:}

Anna L. Lang, Austin M. Krueger, Regina D. Schnegelberger, Brenna R. Kaelin, Maxwell J. Rakutt, Liya Chen, Gavin E Arteel, Juliane I. Rapamycin attenuates liver injury caused by vinyl chloride metabolite chloroethanol and lipopolysaccharide in mice. Beier. Toxicol Appl Pharmacol. 2019 Nov 1; 382: 114745. Published online 2019 Sep6. doi: 10.1016/j.taap.2019.114745

Sears SM, Sharp CN, Krueger A, Oropilla GB, Saforo D, Doll MA, Megyesi J, Beverly LJ, Siskind LJ. C57BL/6 mice require a higher dose of cisplatin to induce renal fibrosis and CCL2 correlates with cisplatin-induced kidney injury. Am J Physiol Renal Physiol. 2020 Oct 1;319(4):F674-F685. doi:

10.1152/ajprenal.00196.2020. Epub 2020 Aug 24. PMID: 32830540; PMCID: PMC7642885.

\section{Service and Outreach:}

2011 - $2016 \quad$ Norton Children's Hospital Volunteer. Summer volunteer program. Primary duties were visiting with patients, cleaning playrooms, and administrative work. 